\title{
GRADINGS OF NON-GRADED HAMILTONIAN LIE ALGEBRAS
}

\author{
A. CARANTI and S. MATTAREI ${ }^{\mathrm{I}-1}$ \\ (Received 20 August 2003; revised 1 April 2005) \\ Communicated by E. A. O'Brien
}

\begin{abstract}
A thin Lie algebra is a Lie algebra graded over the positive integers satisfying a certain narrowness condition. We describe several cyclic grading of the modular Hamiltonian Lie algebras $H\left(2: \mathbf{n} ; \omega_{2}\right)$ (of dimension one less than a power of $p$ ) from which we construct infinite-dimensional thin Lie algebras. In the process we provide an explicit identification of $H\left(2: \mathbf{n} ; \omega_{2}\right)$ with a Block algebra. We also compute its second cohomology group and its derivation algebra (in arbitrary prime characteristic).
\end{abstract}

2000 Mathematics subject classification: primary 17B50; secondary 17B70, 17B56, 17B65.

Keywords and phrases: Modular Lie algebras, graded Lie algebras, derivations, central extensions, loop algebras.

\section{Introduction}

In the last three decades the interest of researchers in finite $p$-groups has increasingly extended to pro-p-groups. This trend was initiated by Leedham-Green and Newman in 1980, who proposed in [45] one way of getting around the universally believed impossibility of a classification of $p$-groups up to isomorphism. One of their intuitions was that of using the coclass rather than the (nilpotency) class of $p$-group as a fundamental invariant. Since the coclass of a group of order $p^{n}$ is defined as the difference between $n$ and the class of the group, this change has no real effect unless one focuses on families of $p$-groups rather than single $p$-groups. A special role is then played by pro- $p$-groups, to which the definition of coclass extends naturally, and which represent entire families of finite $p$-groups as the sets of their finite quotients. In particular, having finite coclass for a pro-p-group $G$ means that all lower central

The authors are grateful to Ministero dell'Istruzione, dell'Università e della Ricerca, Italy, for financial support to the project 'Graded Lie algebras and pro- $p$-groups of finite width'.

(c) 2005 Australian Mathematical Society 1446-7887/05 $\$ A 2.00+0.00$ 
quotients $\gamma_{i}(G) / \gamma_{i+1}(G)$ have order at most $p$ from some point on. The five coclass conjectures advanced in [45] are now theorems thanks to the efforts of several authors, culminating in [42] and [51]. They give information on pro- $p$-groups of finite coclass (Conjecture $\mathrm{C}$, the simplest to state, claiming that every pro- $p$-group of finite coclass is soluble), but also asymptotic information on families of finite $p$-groups of fixed coclass.

Lie-theoretic methods occupy an important place in the theory of $p$-groups, and also in the proofs of the coclass conjectures. The oldest and simplest such method is associating the graded Lie ring $\bigoplus_{i=1}^{\infty} \gamma_{i}(G) / \gamma_{i+1}(G)$ with the lower central series of a (pro-) $p$-group. In many interesting cases the Lie ring is actually a Lie algebra over the field of $p$ elements $\mathbb{F}_{p}$. This approach was already present in disguise in the pioneering work of Blackburn on $p$-groups of coclass one (better known as $p$-groups of maximal class) and the subsequent work of Leedham-Green and McKay (see [43] and the references therein), which inspired the formulation of the coclass conjectures. Lie rings or algebras associated with pro- $p$-groups in this way are graded over the positive integers, and are generated by their component of degree one. The coclass conjectures have natural analogues for graded Lie algebras over an arbitrary field defined by these properties, independently of the connection with pro- $p$-groups, but in this new context all the conjectures turn out to be false. This already occurs in the simplest case of coclass one, as Shalev constructed in [50] the first examples of insoluble graded Lie algebras of maximal class. Here, by a graded Lie algebra of maximal class we mean a Lie algebra $L$ which is graded over the positive integers,

$$
L=\bigoplus_{k=1}^{\infty} L_{k},
$$

where $L_{1}$ has dimension $2, L_{k}$ has dimension 1 for $k>1$, and $L_{k+1}=\left[L_{k}, L_{1}\right]$ for all $k \geq 1$.

Shalev's construction starts from certain finite-dimensional simple modular Lie algebras, originally introduced by Albert and Frank [1], and applies a loop algebra construction (strictly speaking, taking the positive part of a twisted loop algebra, see Subsection 5.1 for details). As a consequence, the resulting graded Lie algebras of maximal class, despite being insoluble, have a kind of periodic structure, in a precise sense. Therefore, one could still hope that each of them is uniquely determined by a suitable finite-dimensional quotient, as is the case with pro- $p$-groups of finite coclass (because each $p$-group of finite coclass $r$ and class large enough, depending on $p$ and $r$, is a quotient of a unique infinite pro- $p$-group of coclass $r$ ). The investigation carried out in [22] showed that this is not the case. In fact, it turned out that most graded Lie algebras of maximal class are not periodic. Nevertheless, Shalev's algebras occupy a unique place in the description of the graded Lie algebras of maximal class 
over fields of odd characteristic, which have been completely classified in [24]. An analogous classification over fields of characteristic two will soon appear in [33].

After the coclass conjectures for pro- $p$-groups were proved, other invariants have been suggested, which may possibly lead to a finer classification of $p$-groups than the rough one provided by the coclass theorems. We do not need the precise definitions of these invariants, which are called width, obliquity and rank, and we refer the interested reader to Chapter 12 of the book [44] by Leedham-Green and McKay. The simplest nontrivial case in terms of these invariants consists of the pro- $p$-groups of width two and obliquity zero. These groups, which do not have finite coclass in general, have also been given the name thin in [15, 14] (originally in the finite case) because of a narrowness characterization in terms of their lattice of (closed) normal subgroups. Some of our terminology, like the diamonds introduced in the next paragraph, originates from that point of view.

An approach to thin groups via the associated graded Lie algebra was taken in [23]. The lower central factors in a thin group are elementary abelian of rank at most two. Those of rank two, in the group or in its associated graded Lie algebra, are called diamonds. There is of course a diamond $G / \gamma_{2}(G)$ on top of a thin group $G$, and if this is the only diamond then $G$ has maximal class. Otherwise, it follows from the theory of $p$-groups of maximal class that in a thin group the second diamond occurs in class at most $p$. In [23] we proved that the associated Lie algebra has bounded dimension, except when the second diamond occurs in class (or degree) 3,5 or $p$. Each of these cases occurs for certain infinite pro- $p$-groups, both $p$-adic analytic and not. For example, Sylow pro- $p$-subgroups of $\operatorname{SL}\left(2, \mathbb{Z}_{p}\right)$ or $\operatorname{SL}\left(2, \mathbb{F}_{p}[[t]]\right)$, or certain 'nonsplit' versions of them, are thin pro- $p$-groups with second diamond in class 3 . A detailed description of these groups in the $p$-adic analytic cases is given in [44, Section 12.2]. Thin pro- $p$-groups with second diamond in class 5 can be realized similarly starting with certain linear groups of type $A_{2}$ over local fields, see [36] or [47]. Finally, the second diamond in class $p$ occurs for one of the wildest known pro- $p$-groups, the Nottingham group (which is thin for $p>2$ ), described in [44, Section 12.4], for example.

A crucial fact for the investigation carried out in [23] was that the condition of a pro-p-group $G$ having obliquity zero can be verified on the associated graded Lie ring, which in this case is a Lie algebra over $\mathbb{F}_{p}$. In this context a more convenient formulation of the condition is the covering property. A graded Lie algebra $L$ as in (1.1), over an arbitrary field and thus not necessarily associated with a group, is called thin if $L_{1}$ has dimension 2 and the following covering property holds:

$$
\text { for all } k \geq 1 \text {, and all } u \in L_{k} \text {, with } u \neq 0 \text {, we have } L_{k+1}=\left[u, L_{1}\right] \text {. }
$$

It follows that $L$ is generated by $L_{1}$ as a Lie algebra and that all homogeneous components have dimension at most two. (See Definition 5.4 and the comments that 
follow for more details.) The arguments of [23] have been extended in $[4,20]$ to show that the second diamond in an infinite-dimensional thin Lie algebra (or one of finite dimension large enough) can only occur in degree $3,5, q$ or $2 q-1$, for some power $q$ of the characteristic $p$ of the underlying field. It follows from [23] that there are, up to isomorphism and with the possible exception of very small characteristics, one or two (depending on the ground field) infinite-dimensional thin Lie algebras with second diamond in degree 3 and no diamond in degree 4 , and one with second diamond in degree 5 . Thus, they are the graded Lie algebras associated with some of the thin pro- $p$-groups mentioned earlier. Machine computations have shown that each of the cases where the second diamond is in degree $q$ or $2 q-1$ splits into a number of subcases, depending on the location of the further diamonds and their types (see Subsection 5.2). Several of these subcases have been investigated in various papers, having in mind a classification of all infinite-dimensional thin Lie algebras as a distant goal. We refer to the paper [21], and to the references mentioned there, for a discussion of thin Lie algebras with second diamond in degree $q$, and to [20,5] for those with second diamond in degree $2 q-1$. Here we restrict ourselves to some general and informal comments on the type of results which have been proved so far.

The results in this subject typically come in pairs, of rather different flavour: namely, a uniqueness theorem and an existence theorem. The former states that a certain initial structure of an infinite-dimensional thin Lie algebra (formulated in terms of the location of the first few diamonds and their types), determines the algebra completely (within the class of thin Lie algebras). More precisely, a certain specified finite-dimensional thin Lie algebra is a quotient of a unique infinite-dimensional thin Lie algebra $L$. This is proved by producing a finite presentation for a central extension $M$ (broadly speaking, the universal central extension of $L$ ). Usually $L$ itself is not finitely presented, see Remark 5.2 for a specific instance of this phenomenon. The existence result consists in the explicit construction of $L$, as a loop algebra of some finite-dimensional Lie algebra $S$, with respect to a suitable cyclic grading, and sometimes with the intervention of an outer derivation of $S$.

The latter type of result brings in an interesting connection with (usually simple) finite-dimensional modular Lie algebras, certain cyclic gradings of them, and their derivations. Their second cohomology group (with values in the trivial module) also plays a role, being closely related with the centre of $M$. Apart from the classical algebras of types $A_{1}$ and $A_{2}$ used in the construction of thin Lie algebras with second diamond in degree 3 and 5 (see $[17,23]$ ), all the remaining cases involve (nonclassical) simple modular Lie algebras of Cartan type, namely Zassenhaus algebras and Hamiltonian algebras of various types. We recall the definitions of these algebras in Sections 2 and 3, and point out in Remark 3.1 other notations in use for them. In particular, infinite-dimensional thin Lie algebras with second diamond in degree $q$ have been constructed as loop algebras of Zassenhaus algebras (which have dimension 
a power of $p$, see $[16,17,18])$, and Hamiltonian algebras of the types $H\left(2: \mathbf{n} ; \omega_{0}\right)=$ $H(2: \mathbf{n})$ (the graded simple Hamiltonian algebras, of dimension two less than a power of $p$, see [3]) and $H\left(2: \mathbf{n} ; \omega_{1}\right)$ (which are Albert-Zassenhaus algebras and have dimension a power of $p$, see [6]).

A preliminary version of the present paper, which predated and inspired some of the other papers cited here, had as its main goal the construction of some infinitedimensional thin Lie algebras with second diamond in degree $2 q-1$ as loop algebras of Hamiltonian algebras $H\left(2: \mathbf{n} ; \omega_{2}\right)$, which have dimension one less than a power of p. (In fact, a construction for those thin Lie algebras had already been given in [20], but as loop algebras of certain finite-dimensional Lie algebras defined 'ad hoc'.) The paper has somehow expanded after we have realised that some of our result may be of interest independently of their application to thin Lie algebras.

Now we describe the contents of this paper in some detail. Our results are presented in Sections 4-8. Sections 2 and 3 are expository and include information on low characteristics which is not easily accessible in the literature. Since much motivation for studying Lie algebras of maximal class and related classes of 'narrow' Lie algebras like thin Lie algebras comes from analogous classes of (pro-) $p$-groups, we have written the expository sections with the aim of making the paper accessible to the group theorist with little knowledge of modular Lie algebras.

We have mentioned earlier the relevance of the second cohomology group (with values in the trivial module) of a finite-dimensional Lie algebra with presentations of the corresponding loop algebra. Therefore, we determine in Section 4 the second cohomology group of the algebras $H\left(2: \mathbf{n} ; \omega_{2}\right)$. We do this according to the classical method used in Farnsteiner [29], which relates the group to the derivations of the algebra, exploiting the presence of a nonsingular associative form. The result is surely well known to experts, but we have been unable to find a suitable reference in the literature. We have also collected in Section 4 known information about derivations and the second cohomology group of $H\left(2: \mathbf{n} ; \omega_{0}\right)$, in all positive characteristics.

In Section 5, we show how various cyclic gradings of $H\left(2: \mathbf{n} ; \omega_{2}\right)$ way, and how they relate to graded Lie algebras of maximal class and to certain thin algebras closely connected to them, which were also studied in [20]. Note that $H\left(2: \mathbf{n} ; \omega_{2}\right)$ has a natural filtration, the standard filtration, inherited by the natural $\mathbb{Z}$-grading of the divided power algebra on which it acts. The filtration is not induced by a grading, however, in contrast to $H\left(2: \mathbf{n} ; \omega_{0}\right)$, which is a graded Lie algebra of Cartan type. (Hence the cheap pun in the title.)

The first manifestation of a connection between 'narrow' infinite-dimensional graded Lie algebras and certain finite-dimensional simple Lie algebras was in Shalev's construction in [50] of insoluble graded Lie algebras of maximal class, which we have mentioned earlier. The finite-dimensional simple algebras of Albert and Frank used by Shalev belong to the larger class of Block algebras, introduced in [11]. It is known 
that Block algebras are Lie algebras of Hamiltonian Cartan type and, in particular, that the algebras of Albert and Frank are Hamiltonian algebras $H\left(2: \mathbf{n} ; \omega_{2}\right)$, at least for $p>3$. (We discuss this further and provide appropriate references towards the end of Section 3.) In Theorem 6.1 we give an explicit isomorphism of the Hamiltonian algebras $H\left(2: \mathbf{n} ; \omega_{2}\right)$ with the algebras of Albert and Frank which is valid in every positive characteristic.

The crucial property of the algebras of Albert and Frank exploited by Shalev in [50] is that they admit a cyclic grading with one-dimensional components, and a nonsingular derivation which permutes them transitively. Benkart, Kostrikin and Kuznetsov proved in [7] and [39] that the only finite-dimensional simple Lie algebras with this property, over an algebraically closed field of characteristic $p>7$, are the Hamiltonian algebras $H\left(2: \mathbf{n} ; \omega_{2}\right)$. The proof rests on the classification of simple modular Lie algebras, which causes the restriction on $p$, but now a classification-free proof of this result for $p>2$ is a consequence of [24]. A more thorough discussion, which includes the case of characteristic two, will be found in Section 6. The cyclic grading of $H\left(2: \mathbf{n} ; \omega_{2}\right)$ and the nonsingular derivation are also the ingredients for discovering the isomorphism with a Block algebra exhibited in Theorem 6.1.

The determination of the second cohomology group of $H\left(2: \mathbf{n} ; \omega_{2}\right)$ in Section 4 depends on a knowledge of its derivation algebra. Since this piece of information is not easily available in low characteristics, we fill this gap by exploiting the other incarnation of $H\left(2: \mathbf{n} ; \omega_{2}\right)$, as originally defined by Albert and Frank. Thus, in Theorem 6.2 we compute the derivation algebra of the algebras of Albert and Frank in arbitrary positive characteristic, by suitably modifying Block's original proof [11], which was valid for $p>3$ only (although for a larger class of algebras).

In [20] we constructed various thin Lie algebras with second diamond in degree $2 q-1$. One can assign a type to each diamond of such algebras, taking values in the underlying field plus $\infty$, in such a way that the locations and types of the diamonds determine the isomorphism type of the algebra (see Subsection 5.2 for more details). The thin Lie algebras with all diamonds of type $\infty$ turn out to be closely connected with graded Lie algebras of maximal class. In particular, there is such a thin Lie algebra associated with each of the graded Lie algebras of maximal class constructed by Shalev in [50]. We describe that in Subsection 5.2 as a loop algebra of $H\left(2: \mathbf{n} ; \omega_{2}\right)$ with respect to a suitable grading. In [20] we also constructed thin Lie algebras with second diamond in degree $2 q-1$ and all diamonds of finite types, as loop algebras of certain Block algebras. The Block algebras used in [20] are actually isomorphic with algebras of Albert and Frank (being simple Block algebras with $G=G_{0}$, see Section 3, and according to known results, for example [13, Lemma 1.8.3]), but were presented there in a different basis. As we have mentioned earlier, the original motivation for the present paper was finding an explicit identification of those algebras with Hamiltonian algebras $H\left(2: \mathbf{n} ; \omega_{2}\right)$ and describing a corresponding cyclic grading 
of the latter. We realize these two tasks in Sections 8 and 7, respectively. We should mention that coexistence of diamonds of both finite and infinite types is possible in the same thin Lie algebra. Such algebras are constructed in [5], as loop algebras of Hamiltonian algebras $H\left(2: \mathbf{n} ; \omega_{2}\right)$ with respect to yet another grading.

\section{Generalities about some Lie algebras of Cartan type}

The Hamiltonian Lie algebras form one of the four families of Lie algebras of Cartan type $W, S, H, K$. Definitions of these families can be found in the recent book of Strade [57]. Here we will limit ourselves to a discussion of the general Lie algebra of Cartan type $W(m: \mathbf{n})$, with a special attention for the Zassenhaus algebra $W(1: n)$, and of two types of Hamiltonian algebras in the next section.

Let $\mathbb{F}$ be a field of prime characteristic $p$, let $\mathbf{n}=\left(n_{1}, \ldots, n_{m}\right)$ be an $m$-tuple of positive integers, and put $n=n_{1}+\cdots+n_{m}$. The algebra of $m$ divided powers truncated at $\mathbf{n}$, denoted by $\mathbb{F}\left[x_{1}, \ldots, x_{m} ; n_{1}, \ldots, n_{m}\right]$ or $\mathbb{F}[m: \mathbf{n}]$ for brevity, is the $\mathbb{F}$-vector space of formal $\mathbb{F}$-linear combinations of monomials $x_{1}^{\left(i_{1}\right)} \cdots x_{m}^{\left(i_{m}\right)}$ with $0 \leq i_{j}<p^{n_{j}}$, with multiplication defined by $x_{j}^{(k)} x_{j}^{(l)}=\left(\begin{array}{c}k+l \\ k\end{array}\right) x_{j}^{(k+l)}$, and extended by linearity and by postulating commutativity and associativity of the multiplication.

Note that, as an algebra, $\mathbb{F}[m: \mathbf{n}]$ is determined up to isomorphism by its dimension $p^{n}$, where $n=n_{1}+\cdots+n_{m}$. In fact, it coincides (up to notation) with the free associative and commutative algebra on the generators $x_{j}^{\left(p^{k_{j}}\right)}$, for $0 \leq j \leq m, 0 \leq$ $k_{j}<n_{j}$, subject to the law $x^{p}=0$ (that is, with $\mathbb{F}\left[x_{1}, \ldots, x_{n}\right] /\left(x_{1}^{p}, \ldots, x_{n}^{p}\right)$ ); this is easily seen by using Lucas' theorem [46] to compute the binomial coefficient $\left(\begin{array}{c}k+l \\ k\end{array}\right)$. In particular, a derivation of $\mathbb{F}[m: \mathbf{n}]$ can be defined by sending the given free generators to arbitrarily chosen elements of $\mathbb{F}[m: \mathbf{n}]$ and extending by the Leibniz rule; furthermore, every derivation is obtained in this way.

However, $\mathbb{F}[m: \mathbf{n}]$ comes equipped with an additional structure, namely a set of divided power maps, which tie the various $p$-(divided) powers of the same variable together [35]. We will not need to know any detail about the divided power maps, except that the definition of special derivations given in [35] or [58] singles out exactly those derivations of $\mathbb{F}[m: \mathbf{n}]$ which are compatible with the divided power maps in a natural sense. It turns out that the special derivations of $\mathbb{F}[m: \mathbf{n}]$ are those of the form $D=f_{1} \partial / \partial x_{1}+\cdots+f_{m} \partial / \partial x_{m}$ with $f_{j} \in \mathbb{F}[m: \mathbf{n}]$, thus acting as $D x_{j}^{(k)}=f_{j} x_{j}^{(k-1)}$ (where $x_{j}^{(-1)}=0$ ).

The Lie algebra of special derivations of $\mathbb{F}[m: \mathbf{n}]$ (which coincides with the full derivation algebra of $\mathbb{F}[m: \mathbf{n}]$ only when $\left.n_{1}=\cdots=n_{m}=1\right)$ is denoted by $W(m: \mathbf{n})$ and is called the general Lie algebra of Cartan type (or generalized Jacobson-Witt algebra). It is simple of dimension $m p^{n}$, unless $m=1$ and $p=2$. A grading of $W(m$ : n) over $\mathbb{Z}^{m}$ is inherited from the natural $\mathbb{Z}^{m}$-grading of $\mathbb{F}[m: \mathbf{n}]$, but it is the grading 
of $\mathbb{F}[m: \mathbf{n}]$ given by total degree of monomials which induces the most important grading of $W(m: \mathbf{n})$, called the standard grading: $W(m: \mathbf{n})=\bigoplus_{i=-1}^{r} L_{i}$, where $L_{i}$ is the subspace spanned by the derivations $x_{1}^{\left(i_{1}\right)} \cdots x_{m}^{\left(i_{m}\right)} \partial / \partial x_{j}$ with $i_{1}+\cdots+i_{m}=i+1$ and $j=1, \ldots, m$ (where $r=p^{n_{1}}+\cdots+p^{n_{m}}-m-1$ ).

REMARK 2.1. What we have described is the unique generalized Jacobson-Witt algebra, for fixed $m$ and $\mathbf{n}$, provided the field $\mathbb{F}$ is algebraically closed; since we have taken $\mathbb{F}$ arbitrary of prime characteristic, $W(m: \mathbf{n})$ is just one of possibly many $\mathbb{F}$-forms of the generalized Jacobson-Witt algebra, see [60], for example. A similar proviso applies to the Hamiltonian algebras which we will describe in the next section, see [49] for a determination of the forms in the restricted case.

In this paper, we will actually only need the Zassenhaus algebras $W(1: n)$, as they occur as distinguished subalgebras of the Hamiltonian algebras which we will consider. In this case, the components of the standard grading are one-dimensional, $L_{i}$ being generated by $E_{i}=x^{(i+1)} d / d x$, for $i=-1, \ldots, r$ (where $r=p^{n}-2$ ). Direct computation shows that

$$
\left[E_{i}, E_{j}\right]=\left(\left(\begin{array}{c}
i+j+1 \\
j
\end{array}\right)-\left(\begin{array}{c}
i+j+1 \\
i
\end{array}\right)\right) E_{i+j}
$$

In particular, we have $\left[E_{-1}, E_{j}\right]=E_{j-1}$, and $\left[E_{0}, E_{j}\right]=j E_{j}$.

The Zassenhaus algebra has also a grading over (the additive group of) $\mathbb{F}_{p^{n}}$, with graded basis consisting of the elements $e_{\alpha}$, for $\alpha \in \mathbb{F}_{p^{n}}$, which satisfy

$$
\left[e_{\alpha}, e_{\beta}\right]=(\beta-\alpha) e_{\alpha+\beta} \text {. }
$$

In particular, note that $\left[e_{0}, e_{\alpha}\right]=\alpha e_{\alpha}$. The bases $\left\{E_{i}\right\}$ and $\left\{e_{\alpha}\right\}$ of $W(1 ; n)$ are sometimes referred to in the literature (at least when $n=1$ ) as a proper basis and a group basis, respectively. One way to obtain the group basis from the proper basis is noting that $E_{-1}+E_{r}$ spans a Cartan subalgebra of $W(1 ; n)$, and computing the corresponding Cartan decomposition. Since ad $\left(E_{-1}+E_{r}\right)$ permutes $E_{r-1}, E_{r-2}, \ldots E_{1}, E_{0}, E_{-1}+2 E_{r}$ cyclically, one quickly finds the formulas

$$
\left\{\begin{array}{l}
e_{0}=E_{-1}+E_{r} \\
e_{\alpha}=E_{r}+\sum_{i=-1}^{r} \alpha^{i+1} E_{i} \quad \text { for } \alpha \in \mathbb{F}_{p^{n}}^{*},
\end{array}\right.
$$

where $r=p^{n}-2$. Note that the first formula is a special case of the second formula if we stipulate that $0^{\circ}=1$.

The inverse formulas are

$$
\left\{\begin{array}{l}
E_{-1}=e_{0}+\sum_{\alpha} e_{\alpha} \\
E_{i}=-\sum_{\alpha} \alpha^{r-i} e_{\alpha} \text { for } i=0, \ldots, r,
\end{array}\right.
$$


where the summations are for $\alpha \in \mathbb{F}_{p^{\prime \prime}}$, and again for the case $i=r$ we understand $\alpha^{0}=1$ for any $\alpha \in \mathbb{F}_{p^{n}}$, whence $E_{r}=-\sum_{\alpha} e_{\alpha}$.

We note in passing that the Zassenhaus algebra is not simple when $p=2$, but has a unique non-trivial ideal, namely $\left\langle E_{i} \mid i \neq r\right\rangle=\left\langle e_{\alpha} \mid \alpha \neq 0\right\rangle$, which we will refer to as the simple Zassenhaus algebra. The above transition formulas are clearly valid in this case, too. As we will point out in the next section, a simple Zassenhaus algebra in characteristic two can also be regarded as a Hamiltonian algebra (and, in turn, as a Block algebra).

Since the problem of inverting formulas similar to those which relate the bases $\left\{e_{\alpha}\right\}$ and $\left\{E_{i}\right\}$ of the Zassenhaus algebra will occur repeatedly in this paper, we record the solution explicitly. To simplify notation and computations it will be useful to set $0^{0}=1$ once and for all. The customary rules $\alpha^{i} \beta^{i}=(\alpha \beta)^{i}$ and $\alpha^{i} \alpha^{j}=\alpha^{i+j}$ now hold for $\alpha, \beta$ in a field and $i, j$ non-negative integers. Note that with this convention the expression $\sum_{\alpha \in \mathbb{F}_{q}} \alpha^{j}$, where $\mathbb{F}_{q}$ is the finite field of $q$ elements, becomes meaningful for every non-negative integer $j$; its value is -1 if $j$ is a positive multiple of $q-1$, and 0 otherwise.

LEMMA 2.2. The linear relations $a_{\alpha}=\sum_{j=0}^{q-1} \alpha^{j} b_{j}$, for $\alpha \in \mathbb{F}_{q}$, between elements $a_{\alpha}\left(\alpha \in \mathbb{F}_{q}\right)$ and $b_{j}(j=0, \ldots, q-1)$ of any vector space over $\mathbb{F}_{q}$, are equivalent to the relations

$$
\left\{\begin{array}{l}
b_{0}=a_{0}, \\
b_{j}=-\sum_{\alpha \in \mathbb{F}_{q}} \alpha^{q-1-j} a_{\alpha}, \quad \text { for } j=1, \ldots, q-1 .
\end{array}\right.
$$

PROOF. If $\omega$ is a primitive $n$-th root of unity in any field, then the sets of formulas

$$
a_{i}=\sum_{j=1}^{n} \omega^{i j} b_{j} \quad \text { and } \quad b_{j}=\frac{1}{n} \sum_{i=1}^{n} \omega^{-i j} a_{i},
$$

relating subsets $\left\{a_{i} \mid i=1, \ldots, n\right\}$ and $\left\{b_{j} \mid j=1, \ldots, n\right\}$ of any vector space over that field, are inverse of each other. This is an instance of a Fourier transform and its inverse over a cyclic group of order $n$, and can be easily proved using the fact that $\sum_{j=1}^{n} \omega^{i j}$ equals $n$ if $i$ is a multiple of $n$, and 0 otherwise.

In particular, taking as $\omega$ a generator of $\mathbb{F}_{q}^{*}$, we obtain that the sets of formulas

$$
a_{\alpha}=\sum_{j=1}^{q-1} \alpha^{j} b_{j} \quad \text { and } \quad b_{j}=-\sum_{\alpha \in \mathbb{F}_{q}^{*}} \alpha^{-j} a_{\alpha},
$$

which relate elements $a_{\alpha}\left(\alpha \in \mathbb{F}_{q}\right)$ and $b_{j}(j=0, \ldots, q-1)$ of any vector space over $\mathbb{F}_{q}$, are inverse of each other. 
Now consider the formulas $a_{\alpha}=\sum_{j=0}^{q-1} \alpha^{j} b_{j}$ for $\alpha \in \mathbb{F}_{q}$, note that one of them is $a_{0}=b_{0}$, and write the remaining ones in the form $a_{\alpha}-a_{0}=\sum_{j=1}^{q-1} \alpha^{j} b_{j}$. As we have seen above, these formulas can be inverted, and yield

$$
b_{j}=-\sum_{\alpha \in \mathbb{F}_{i}^{*}} \alpha^{-j}\left(a_{\alpha}-a_{0}\right)=-\delta_{j, q-1} a_{0}-\sum_{\alpha \in \mathbb{F}_{\dot{q}}} \alpha^{-j} a_{\alpha}=-\sum_{\alpha \in \mathbb{F}_{q}} \alpha^{q-1-j} a_{\alpha},
$$

for $j=1, \ldots, q-1$.

\section{Hamiltonian algebras and Block algebras}

The Lie algebras of Cartan type $S, H$ and $K$ are defined as subalgebras of the generalized Jacobson-Witt algebra $W(m: \mathbf{n})$, and depend on a choice of a certain differential form $\omega$ (or, equivalently, of a certain automorphism of $W(m: \mathbf{n})$ ). For this to make sense in general one must complete the algebra of divided powers $\mathbb{F}[m: \mathbf{n}]$ to an algebra of divided power series. However, this will not be necessary to define the only Hamiltonian algebras which we will consider in this paper, namely $H\left(2: \mathbf{n} ; \omega_{j}\right)$ for $j=0$ or 2 . Note that the Hamiltonian algebras in two variables can also be considered as belonging to the Cartan series of special algebras (and thus be denoted by $S\left(2: \mathbf{n} ; \omega_{j}\right)$ ). A rather condensed description of all four classes of Lie algebras of Cartan type, but complete with all the relevant references, can be found in [7], to which we also conform our notation. (See Remark 3.1 concerning our notation.) For a more extensive discussion see [57].

As in the previous section, we assume only that $\mathbb{F}$ is a field of prime characteristic $p$, and point out which statements need restrictions on $\mathbb{F}$ as we go along. Let $\mathbb{F}[2: \mathbf{n}]=\mathbb{F}\left[x, y ; n_{1}, n_{2}\right]$ be the algebra of divided powers in two variables $x, y$ of heights $\mathbf{n}=\left(n_{1}, n_{2}\right)$. It will be convenient to put $\bar{x}=x^{\left(p^{n_{1}}-1\right)}, \bar{y}=y^{\left(p^{n_{2}}-1\right)}$, and $e=\bar{x} \bar{y}$. Then $H\left(2: \mathbf{n} ; \omega_{j}\right)$ can be defined as the second derived algebra of

$$
\tilde{H}\left(2: \mathbf{n} ; \omega_{j}\right)=\left\{D \in W(2: \mathbf{n}) \mid D \omega_{j}=0\right\},
$$

where $\omega_{0}=d x \wedge d y$ and $\omega_{2}=(1-e) d x \wedge d y$. Note that the (formal) differential forms here are simply elements of the exterior algebra on the set $\{d x, d y\}$ over $\mathbb{F}[2: \mathbf{n}]$. In particular, the space of differential 2 -forms is the free $\mathbb{F}[2: \mathbf{n}]$-module on the basis $\{d x \wedge d y\}$, and is a $W(2: \mathbf{n})$-module via

$$
D(f d x \wedge d y)=(D f) d x \wedge d y+f d(D x) \wedge d y+f d x \wedge d(D y)
$$

for $D \in W(2: \mathbf{n})$, where $d f=(\partial f / \partial x) d x+(\partial f / \partial y) d y$. When dealing with derivations of $H\left(2: \mathbf{n} ; \omega_{j}\right)$ it will be useful to consider the larger algebra

$$
C \tilde{H}\left(2: \mathbf{n} ; \omega_{j}\right)=\left\{D \in W(2: \mathbf{n}) \mid D \omega_{j}=c \omega_{j}, c \in \mathbb{F}\right\},
$$


which contains $\tilde{H}\left(2: \mathbf{n} ; \omega_{j}\right)$ as an ideal of codimension one and zero for $j=0,2$, respectively.

REMARK 3.1. We should mention that the notation for the Lie algebras of Cartan type is not uniform in the literature. In particular, $H(-)$ sometimes denotes what we have indicated with $\tilde{H}(-)$ here, and so one has to take the (first or) second derived algebra $H(-)^{(2)}$ to obtain the simple algebra. Furthermore, special, Hamiltonian and contact algebras can also be obtained as subalgebras of the generalized JacobsonWitt algebra (on an algebra of divided power series, in general) by means of certain automorphisms $\Phi$, instead of differential forms $\omega$. For example, our $H\left(2: \mathbf{n} ; \omega_{0}\right)$ and $H\left(2: \mathbf{n} ; \omega_{2}\right)$ are denoted by $H(2 ; \underline{n})^{(2)}=H(2 ; \underline{n} ; \text { id })^{(2)}$ and $H(2 ; \underline{n} ; \Phi(\tau))^{(1)}$ in the book [57], and similarly in many papers. We also note that, strictly speaking, the notation $H\left(2: \mathbf{n} ; \omega_{i}\right)$ which we use here would be only justified when working over an algebraically closed field (and of characteristic large enough). This is because only in that case it can be shown that any form $\omega$ defining a Hamiltonian algebra can be assumed to have certain specific forms $\omega_{0}, \omega_{1}, \omega_{2}$ (see [7] and the reference therein for the most general results, but [61, Corollary 2] suffices for the Hamiltonian algebras $H\left(2: \mathbf{n} ; \omega_{i}\right)$ under consideration here). Whenever we consider $H\left(2: \mathbf{n} ; \omega_{i}\right)$ over an arbitrary field in this paper, we refer to the specific form defined above.

Since the space of differential 2-forms on $\mathbb{F}[2: \mathbf{n}]$ has a natural structure of graded module for the $\mathbb{Z}^{2}$-graded Lie algebra $W(2: \mathbf{n})$, and $\omega_{0}$ is a homogeneous element with respect to this grading, $\tilde{H}\left(2: \mathbf{n} ; \omega_{0}\right)$ and $C \tilde{H}\left(2: \mathbf{n} ; \omega_{0}\right)$ are graded subalgebras of $W(2: \mathbf{n})$ with respect to the $\mathbb{Z}^{2}$-grading. In particular, they are also graded subalgebras with respect to the standard grading of $W(2: \mathbf{n})$, and thus they acquire what is called their standard grading. They are usually referred to as the graded Lie algebras of Hamiltonian type, in contrast to their relatives with respect to forms of type $\omega_{1}$ and $\omega_{2}$, which are only filtered.

Thus, in determining an explicit expression for the generic element $D$ of these subalgebras one may assume that $D$ is homogeneous with respect to the $\mathbb{Z}^{2}$-grading of $W(2: \mathbf{n})$. A simple computation as in [40, pages $255-257$ ], or [58, page 162ff.] (where the assumption that $p>2$ is not used before Theorem 4.5) shows that $\tilde{H}(2$ : $\left.\mathbf{n} ; \omega_{0}\right)$ consists of the derivations of $\mathbb{F}[2: \mathbf{n}]$ of the form $\mathscr{D}_{H}(f)=f_{y} \partial / \partial x-f_{x} \partial / \partial y$ for some $f \in \tilde{P}\left(2: \mathbf{n} ; \omega_{0}\right)=\mathbb{F}[2: \mathbf{n}] \oplus\left\langle x^{\left(p^{n_{1}}\right)}, y^{\left(p^{n_{2}}\right)}\right\rangle$ (where $f_{x}$ and $f_{y}$ stand for $\partial f / \partial x$ and $\partial f / \partial y$, respectively), and that $C \tilde{H}\left(2: \mathbf{n} ; \omega_{0}\right)=\tilde{H}\left(2: \mathbf{n} ; \omega_{0}\right) \oplus\langle x \partial / \partial x\rangle$. The latter can be written in the more symmetric form $\tilde{H}\left(2: \mathbf{n} ; \omega_{0}\right) \oplus\langle x \partial / \partial x+y \partial / \partial y\rangle$ if $p>2$. Since

$$
\begin{aligned}
{\left[\mathscr{D}_{H}(f), \mathscr{D}_{H}(g)\right] } & =\left(f_{y} g_{x}-f_{x} g_{y}\right)_{y} \frac{\partial}{\partial x}-\left(f_{y} g_{x}-f_{x} g_{y}\right)_{x} \frac{\partial}{\partial y} \\
& =\mathscr{D}_{H}\left(\mathscr{D}_{H}(f)(g)\right),
\end{aligned}
$$


the map $\mathscr{D}_{H}$ is a homomorphism from the $\tilde{H}\left(2: \mathbf{n} ; \omega_{0}\right)$-module $\tilde{P}\left(2: \mathbf{n} ; \omega_{0}\right)$ onto the adjoint module for $\tilde{H}\left(2: \mathbf{n} ; \omega_{0}\right)$, with kernel $\langle 1\rangle$. The associative (and commutative) algebra $\tilde{P}\left(2: \mathbf{n} ; \omega_{0}\right)$ can be endowed with an additional structure of Lie algebra with respect to the Poisson bracket $\{f, g\}=\mathscr{D}_{H}(f)(g)=f_{y} g_{x}-f_{x} g_{y}$, and the map $\mathscr{D}_{H}$ yields a Lie algebra isomorphism from $\tilde{P}\left(2: \mathbf{n} ; \omega_{0}\right) /\langle 1\rangle$ onto $\tilde{H}\left(2: \mathbf{n} ; \omega_{0}\right)$. (Note that our notation for the map $\mathscr{D}_{H}$ and, consequently, for the Poisson bracket, differs in sign from that of [58], and agrees with [37] or [8] instead.) Under this isomorphism, the second derived subalgebra $H\left(2: \mathbf{n} ; \omega_{0}\right)$ of $\tilde{H}\left(2: \mathbf{n} ; \omega_{0}\right)$ corresponds to the subalgebra

$$
P\left(2: \mathbf{n} ; \omega_{0}\right) /\langle 1\rangle=\left\langle x^{(i)} y^{(j)} \in \mathbb{F}[2: \mathbf{n}] \mid x^{(i)} y^{(j)} \neq e\right\rangle /\langle 1\rangle,
$$

of dimension $p^{n}-2$, where $n=n_{1}+n_{2}$. This is a simple Lie algebra if $p>2$, see [40] or [58]. In characteristic two it is simple provided $n_{1}>1$ and $n_{2}>1$, as one can prove along the lines of [58, Theorem 3.5 or Theorem 4.5 of Chapter 4]. However, $P\left(2:\left(1, n_{2}\right) ; \omega_{0}\right) /\langle 1\rangle$ has $\left\langle y^{(j)} \mid 0 \leq j<2^{n_{2}}\right\rangle /\langle 1\rangle$ as an ideal. In fact, it is the split extension of a simple Zassenhaus algebra by its adjoint module. It can also be viewed as the tensor product of a simple Zassenhaus algebra with the algebra of divided powers $\mathbb{F}[z: 1]$.

Since the Lie algebra homomorphism $\mathscr{D}_{H}$ is also a homomorphism of $\tilde{H}(2$ : $\left.\mathbf{n} ; \omega_{0}\right)$-modules, the action of ad $\mathscr{D}_{H}(f)$ as an inner derivation of the Lie algebra structure of $\tilde{P}\left(2: \mathbf{n} ; \omega_{0}\right)$ coincides with the action of $\mathscr{D}_{H}(f)$ as a derivation of the associative algebra structure of $\tilde{P}\left(2: \mathbf{n} ; \omega_{0}\right)$. This will be useful when computing with derivations of $H\left(2: \mathbf{n} ; \omega_{0}\right)$ in Sections 4 and 5 . For this reason we will simply regard $\mathscr{D}_{H}(f)$ as a derivation of the Lie algebra $\tilde{H}\left(2: \mathbf{n} ; \omega_{0}\right)$ (rather than the more cumbersome notation ad $\mathscr{D}_{H}(f)$ ). A word of caution, however: this does not extend to derivations of $\tilde{P}\left(2: \mathbf{n} ; \omega_{0}\right)$ which are not inner. In fact, $\mathscr{D}_{H}$ is not a homomorphism of $C \tilde{H}\left(2: \mathbf{n} ; \omega_{0}\right)$-modules, because $\left[D, \mathscr{D}_{H}\left(x^{(i)} y^{(j)}\right)\right]=(i+j-2) \mathscr{D}_{H}\left(x^{(i)} y^{(j)}\right)$ while $D\left(x^{(i)} y^{(j)}\right)=(i+j) x^{(i)} y^{(j)}$, for $D=x \partial / \partial x+y \partial / \partial y$.

In this paper, we will find convenient to always talk about $\tilde{H}\left(2: \mathbf{n} ; \omega_{0}\right)$ while actually carrying out explicit computations inside $\tilde{P}\left(2: \mathbf{n} ; \omega_{0}\right) /\langle 1\rangle$ with the Poisson bracket (and similarly for $\tilde{H}\left(2: \mathbf{n} ; \omega_{2}\right)$, later). Writing $x^{(i)} y^{(j)}$ for $x^{(i)} y^{(j)}+\langle 1\rangle$, we have

$$
\begin{aligned}
\left\{x^{(i)} y^{(j)}, x^{(k)} y^{(l)}\right\} & =x^{(i)} y^{(j-1)} x^{(k-1)} y^{(l)}-x^{(i-1)} y^{(j)} x^{(k)} y^{(l-1)} \\
& =N(i, j, k, l) x^{(i+k-1)} y^{(j+l-1)},
\end{aligned}
$$

where

$$
N(i, j, k, l)=\left(\begin{array}{c}
i+k-1 \\
i
\end{array}\right)\left(\begin{array}{c}
j+l-1 \\
j-1
\end{array}\right)-\left(\begin{array}{c}
i+k-1 \\
i-1
\end{array}\right)\left(\begin{array}{c}
j+l-1 \\
j
\end{array}\right) .
$$

The $\mathbb{Z}^{2}$-grading of $L=\tilde{H}\left(2: \mathbf{n} ; \omega_{0}\right)$ is $L=\bigoplus_{(i, j) \in \mathbb{Z}^{2}} L_{i, j}$, where $L_{i, j}=\left\langle x^{(i+1)} y^{(j+1)}\right\rangle$ in the Poisson bracket notation, and the standard grading is $L=\bigoplus_{k \in \mathbb{Z}} \bar{L}_{k}$, where 
$\bar{L}_{k}=\sum_{i+j=k} L_{i, j}$ consists of all homogeneous polynomials of degree $k+2$. Note that $\vec{L}_{k}$ is trivial unless $-1 \leq k \leq p^{n_{1}}+p^{n_{2}}-4$ (or unless $-1 \leq k \leq p^{n_{1}}+p^{n_{2}}-5$, if we restrict our attention to $\left.H\left(2: \mathbf{n} ; \omega_{0}\right)\right)$.

We consider now $C \tilde{H}\left(2: \mathbf{n} ; \omega_{2}\right)$. Although the form $\omega_{2}$ is not homogeneous with respect to the $\mathbb{Z}^{2}$-grading, it becomes so with respect to the grading obtained by viewing it modulo $\left(p^{n_{1}}-1, p^{n_{2}}-1\right)$. Thus, $C \tilde{H}\left(2: \mathbf{n} ; \omega_{2}\right)$ is a graded subalgebra of $W(2: \mathbf{n})$ with respect to its $A$-grading, where $A=\mathbb{Z}^{2} /\left\langle\left(p^{n_{1}}-1, p^{n_{2}}-1\right)\right\rangle$. We will examine some specializations of this grading in Section 5. Consideration of $A$-homogeneous elements makes it straightforward to determine an explicit form for the elements of $C \tilde{H}\left(2: \mathbf{n} ; \omega_{2}\right)$. One finds that $\tilde{H}\left(2: \mathbf{n} ; \omega_{2}\right)$ coincides with $C \tilde{H}\left(2: \mathbf{n} ; \omega_{2}\right)$, and can be identified with $\tilde{P}\left(2: \mathbf{n} ; \omega_{2}\right) /\langle\mathbf{1}\rangle=\left(\mathbb{F}[2: \mathbf{n}] \oplus\left\langle x^{\left(p^{\left.n_{1}\right)}\right.}, y^{\left(p^{n_{2}}\right)}\right\rangle\right) /\langle 1\rangle$ with the Poisson bracket $\{f, g\}=(1+e)\left(f_{y} g_{x}-f_{x} g_{y}\right)$. The derived subalgebra $H\left(2: \mathbf{n} ; \omega_{2}\right)$ of $\tilde{H}(2:$ $\left.\mathbf{n} ; \omega_{2}\right)$ has dimension $p^{n}-1$ and corresponds to $\mathbb{F}[2: \mathbf{n}] /\langle 1\rangle$ with the Poisson bracket. It is simple (in every characteristic), as will follow from its identification, given in Section 6, with a certain Block algebra, whose simplicity was proved in [11]. In characteristic two $H\left(2:(1, n) ; \omega_{2}\right)$ is isomorphic with the simple Zassenhaus algebra of dimension $2^{n+1}-1$, an isomorphism being obtained by mapping $x y^{(j)} \mapsto E_{j-1}$ and $y^{(j)} \mapsto E_{j+2^{n}-2}$. A curious consequence of this isomorphism is that in characteristic two $H\left(2:(1, n) ; \omega_{2}\right)$ can be embedded in $H\left(2:(1, n+1) ; \omega_{2}\right)$ as a subalgebra, namely as the simple Zassenhaus subalgebra $\left\langle x y^{(j)}: j=0, \ldots, p^{n+1}-2\right\rangle$.

The Poisson bracket of monomials

$$
\left\{x^{(i)} y^{(j)}, x^{(k)} y^{(l)}\right\}=(1+e) N(i, j, k, l) x^{(i+k-1)} y^{(j+l-1)}
$$

for $\tilde{H}\left(2: \mathbf{n} ; \omega_{2}\right)$ coincides with that for $\tilde{H}\left(2: \mathbf{n} ; \omega_{0}\right)$ except for the products $\{y, x\}=$ $-\{x, y\}=e$. This shows that the $A$-grading of $\tilde{H}\left(2: \mathbf{n} ; \omega_{2}\right)$ cannot be lifted to a $\mathbb{Z}^{2}$-grading. For the same reason, $\tilde{H}\left(2: \mathbf{n} ; \omega_{2}\right)$ is not $\mathbb{Z}$-graded by the subspaces $\bar{L}_{k}=$ $\sum_{i+j=k} L_{i, j}$ defined as before. However, it is filtered by the subspaces $\bar{L}^{k}=\sum_{h \geq k} \bar{L}_{h}$, that is, $L=\bar{L}^{-1} \supseteq \bar{L}^{0} \supseteq \bar{L}^{1} \supseteq \cdots$ (where $\bar{L}^{k}=0$ for $k>p^{n_{1}}+p^{n_{2}}-4$ ), and $\left[\bar{L}^{h}, \bar{L}^{k}\right] \subseteq \bar{L}^{h+k}$. This is called the standard filtration of $\tilde{H}\left(2: \mathbf{n} ; \omega_{2}\right)$, and the graded Lie algebra associated with it is $\tilde{H}\left(2: \mathbf{n} ; \omega_{0}\right)$.

We should note here that both Hamiltonian algebras $H\left(2: \mathbf{n} ; \omega_{0}\right)$ and $H\left(2: \mathbf{n} ; \omega_{2}\right)$ were originally constructed in a different way. In fact, after being introduced first in [1] among other examples, they became special cases of a more general construction due to Block [11]. We briefly recall only a special case of Block's construction which is relevant to the present paper, and we refer to [11] or [48, page 110] for full generality.

Let $G$ be an elementary abelian $p$-group of order $p^{n}$, written additively, let $\delta \in G$, and let $f: G \times G \rightarrow G$ be a non-singular biadditive function of the form $f(\alpha, \beta)=$ $g(\alpha) h(\beta)-g(\beta) h(\alpha)$ for some additive functions $g, h: G \rightarrow G$. A vector space $L$ over a field $F$ of characteristic $p$, with basis $\left\{u_{\alpha} \mid \alpha \in G\right\}$ in bijective correspondence with the elements of $G$, becomes a Lie algebra by defining a multiplication on the 
basis elements via $\left[u_{\alpha}, u_{\beta}\right]=f(\alpha, \beta) u_{\alpha+\beta-\delta}$ and extending linearly. The element $u_{0}$ is central in $L$, and the elements $u_{\alpha}$ with $\alpha \neq \delta$ span an ideal of $L$. If $\delta=0$ the ideal $\left\langle u_{\alpha} \mid \alpha \neq 0\right\rangle$ is a simple Lie algebra, and if $\delta \neq 0$ the quotient $\left\langle u_{\alpha} \mid \alpha \neq \delta\right\rangle /\left\langle u_{0}\right\rangle$ is simple. In both cases the simple algebra is called a Block algebra. (These special cases of Block's construction had already been introduced by Albert and Frank in [1], and denoted by $\mathscr{L}_{0}$ and $\mathscr{L}_{\delta}$ there; in this paper we refer to the algebras $\mathscr{L}_{\delta}$ with $\delta \neq 0$ as algebras of Albert and Frank, conforming to $[22,24,50]$.

It is known that if $\mathbb{F}$ is algebraically closed of characteristic $p>3$, the above special cases of Block's construction yield exactly the Hamiltonian algebras $H\left(2: \mathbf{n} ; \omega_{2}\right)$ if $\delta=0$, and the algebras $H\left(2: \mathbf{n} ; \omega_{0}\right)$ if $\delta \neq 0$. For example, this is stated in [13, Lemma 1.8.3] under the blanket assumption of that paper that $p>7$, but the proof given there is seen to be valid for $p>3$. (In particular, one ingredient of that proof, namely [61, Corollary 2], was originally proved for $p>5$; however, it is now a special case of more general results in [9] or [54] which assume only $p>3$.)

Note that the method of proof of [13, Lemma 1.8.3] does not easily produce explicit realizations of $H\left(2: \mathbf{n} ; \omega_{0}\right)$ and $H\left(2: \mathbf{n} ; \omega_{2}\right)$ (with respect to the given forms) as Block algebras. In fact, in essence (using automorphisms $\Phi$ rather than forms $\omega$ ), it shows that for appropriate choices of the form $\omega$ the Hamiltonian algebra $H(2: \mathbf{n} ; \omega)$ is a Block algebra of dimension $p^{n}-2$ or $p^{n}-1$, and then appeals to [13, Theorem 1.8.1] (which quotes [61, Corollary 2]) to conclude that $H(2: \mathbf{n} ; \omega) \cong H\left(2: \mathbf{n} ; \omega_{i}\right)$ for $i=0$ or 2 , respectively. In Section 6 of the present paper we do give an explicit realization of $H\left(2: \mathbf{n} ; \omega_{2}\right)$ as a Block algebra, and we do that for arbitrary prime characteristic $p$ (thus including 2 and 3). We mention that, more generally, it was announced in [38] and proved in [39] that every algebra $H\left(m: \mathbf{n} ; \omega_{2}\right)$ is a Block algebra.

\section{The second cohomology group of $H\left(2: n ; \omega_{2}\right)$}

In this section we appeal to some results which were formulated under the assumption that the ground field is algebraically closed, or at least perfect; since derivations and cohomology are essentially independent of the ground field, these assumptions are immaterial here in view of Remark 3.1. We assume that the ground field has odd characteristic. At some stage in the discussion we also need to assume that the characteristic $p$ is greater than three (see Remark 4.3 for the case $p=3$ ), but our main result, Theorem 4.2 , does not depend on this assumption. Finally, we deal with the case of characteristic two in Remarks 4.4 and 4.5.

The dimensions of the second cohomology groups $H^{2}(L, \mathbb{F})$ of some graded Lie algebras of Cartan type with values in the trivial module were computed in [29] (but see also [26]). In particular, according to [29, Theorem 2.4], $H^{2}(L, \mathbb{F})$ has dimension $n_{1}+n_{2}+1$ for the graded Hamiltonian algebra $L=H\left(2: \mathbf{n} ; \omega_{0}\right)$. Here we compute 
$H^{2}(L, \mathbb{F})$ for $L=H\left(2: \mathbf{n} ; \omega_{2}\right)$ and show that it has dimension $n_{1}+n_{2}$. (In the special case $\mathbf{n}=(1,1)$ this can essentially be found in [55, Theorem 6.3].)

Following [29], we briefly recall the classical method (see [48, page 102]) employed there to compute $H^{2}(L, \mathbb{F})$ from the space of outer derivations of $L$, in presence of a nondegenerate associative form on $L$. In addition, we exhibit a basis of $H^{2}(L, \mathbb{F})$ for $L=H\left(2: \mathbf{n} ; \omega_{0}\right)$. According to [29, Proposition 1.3], for any Lie algebra $L$ over a field $\mathbb{F}$ there is an injective homomorphism $H^{2}(L, \mathbb{F}) \rightarrow H^{1}\left(L, L^{*}\right)$, where $L^{*}$ denotes the dual of the adjoint module of $L$. This monomorphism is induced by the map $\varphi \mapsto D_{\varphi}$ which sends a 2-cocycle $\varphi \in Z^{2}(L, \mathbb{F})$ to the derivation $D_{\varphi}: L \rightarrow L^{*}$ with $D_{\varphi}(\xi)=\varphi(\xi, \cdot)$. Furthermore, the image of the monomorphism consists of the cohomology classes represented by skew derivations, that is to say, derivations $D: L \rightarrow L^{*}$ which satisfy $D(\xi)(\eta)=-D(\eta)(\xi)$, for all $\xi, \eta \in L$.

Now assume that $L$ possesses a nondegenerate associative form $\lambda$, that is, a symmetric bilinear form $\lambda: L \times L \rightarrow \mathbb{F}$ satisfying $\lambda([\xi, \eta], \theta)=\lambda(\xi,[\eta, \theta])$ for all $\xi, \eta, \theta \in L$. (Note that the latter condition together with anticommutativity of the Lie bracket easily implies that $\lambda([\xi, \eta], \theta)=\lambda(\theta,[\xi, \eta])$, hence the symmetry of $\lambda$ is automatic if $L$ is perfect. In particular, in view of the interpretation of associativity which we are about to give, there are non nonzero $L$-module homomorphisms $L \wedge L \rightarrow \mathbb{F}$ if $L$ is perfect, in odd characteristic.) Since the associativity condition can be written in the equivalent form $\lambda([\eta, \xi], \theta)+\lambda(\xi,[\eta, \theta])=0$, it simply means that the corresponding linear map $\lambda: L \otimes L \rightarrow \mathbb{F}$ is a homomorphism of $L$-modules into the trivial module. Consequently, the adjoint module of $L$ is self-dual; this condition is, in fact, equivalent with the existence of a nondegenerate bilinear form on $L$ satisfying associativity but not necessarily symmetric.

By composition with the inverse of the $L$-module isomorphism $L \rightarrow L^{*}$ given by $\xi \mapsto \lambda(\xi, \cdot)$, the monomorphism $H^{2}(L, \mathbb{F}) \rightarrow H^{1}\left(L, L^{*}\right)$ turns into a monomorphism $H^{2}(L, \mathbb{F}) \rightarrow H^{1}(L, L)=\operatorname{Der}(L) / \operatorname{ad}(L)$, where $\operatorname{ad}(L)$ is the space of inner derivations of $L$. Its image is $\operatorname{SkDer}(L) / \operatorname{ad}(L)$, where $\operatorname{SkDer}(L)$ denotes the space of all derivations $D: L \rightarrow L$ which are skew with respect to the associative form $\lambda$, that is, which satisfy $\lambda(D(\xi), \eta)=-\lambda(D(\eta), \xi$ ) for all $\xi, \eta \in L$ (see [29, Remark after Proposition 1.3]). Writing this condition in the equivalent form $\lambda(D(\xi), \eta)+\lambda(\xi, D(\eta))=0$ shows that a derivation $D$ of $L$ is skew exactly if $D$ annihilates the form $\lambda$ viewed as an element of $(L \otimes L)^{*}$, the dual of the tensor square of the adjoint module of $L$; it follows, in particular, that $\operatorname{SkDer}(L)$ is a $p$-subalgebra of $\operatorname{Der}(L)$ containing all inner derivations of $L$.

The isomorphism $H^{2}(L, \mathbb{F}) \rightarrow \operatorname{SkDer}(L) / \operatorname{ad}(L)$ is actually induced by an isomorphism $Z^{2}(L, \mathbb{F}) \rightarrow \operatorname{SkDer}(L)$, which we describe here for convenience. Because of the nondegeneracy of $\lambda$, for each cocycle $\varphi \in Z^{2}(L, \mathbb{F})$ there is a unique derivation 
$D_{\varphi}: L \rightarrow L$, necessarily skew, such that

$$
\lambda\left(D_{\varphi}(\xi), \eta\right)=\varphi(\xi, \eta) \quad \text { for all } \xi, \eta \in L .
$$

Conversely, the 2-cocycle associated with the skew derivation $D: L \rightarrow L$ is given by

$$
\varphi(D)(\xi, \eta)=\lambda(D(\xi), \eta) \quad \text { for all } \xi, \eta \in L .
$$

Now we apply these well-known facts to the Hamiltonian algebras under consideration (the case of $H\left(2: \mathbf{n} ; \omega_{0}\right)$ being already dealt with in [29]). It is known from [28, Theorem 4.4] or [58, Chapter 4, Theorem 6.5] that the graded algebra $H\left(2: \mathbf{n} ; \omega_{0}\right)$ has a non-degenerate associative form $\lambda$, which in our notation becomes

$$
\lambda\left(x^{(i)} y^{(j)}, x^{(k)} y^{(l)}\right)=(-1)^{i+j} \delta\left(i+k, p^{n_{1}}-1\right) \delta\left(j+l, p^{n_{2}}-1\right) .
$$

Thus the dual basis of $\left\{x^{(i)} y^{(j)}\right\}$ with respect to the nondegenerate form $\lambda$ is given by $\left(x^{(i)} y^{(j)}\right)^{*}=(-1)^{i+j} x^{\left(p^{n_{1}}-1-i\right)} y^{\left(p^{n_{2}}-1-j\right)}$.

We assume now that $p>3$. The derivation algebras of the simple Lie algebras of Cartan type are known and are summarized in [7, pages 903-905]. (Alternatively, the derivation algebras of Block algebras, which include the Hamiltonian algebras under consideration here, were already computed in [11, Theorem 14], again for $p>3$.) In particular, it is known that

$$
\operatorname{Der} H\left(2: \mathbf{n} ; \omega_{i}\right)=\overline{C \tilde{H}\left(2: \mathbf{n} ; \omega_{i}\right)},
$$

the $p$-closure of $C \tilde{H}\left(2: \mathbf{n} ; \omega_{i}\right)$ in Der $\mathbb{F}[2: \mathbf{n}]$, for $i=0,2$. More explicitly, a basis for the space of outer derivations $L=H\left(2: \mathbf{n} ; \omega_{0}\right)$ (or, more precisely, a set of representatives for a basis of $\left.H^{3}(L, L)=\operatorname{Der}(L) / \operatorname{ad}(L)\right)$ consisting of homogeneous derivations with respect to the $\mathbb{Z}^{2}$-grading is as follows:

(1) $(\operatorname{ad} x)^{p^{r}}$, of degree $\left(0,-p^{r}\right)$, for $0<r<n_{2}$;

(2) $(\operatorname{ad} y)^{p^{s}}$, of degree $\left(-p^{s}, 0\right)$, for $0<s<n_{1}$;

(3) $\operatorname{ad}\left(x^{\left(p^{n_{1}}\right)}\right)$, of degree $\left(p^{n_{1}}-1,-1\right)$;

(4) $\operatorname{ad}\left(y^{\left(p^{\left.n_{2}\right)}\right)}\right)$, of degree $\left(-1, p^{n_{2}}-1\right)$;

(5) $\operatorname{ad}(\bar{x} \bar{y})=\left[\operatorname{ad}\left(x^{\left(p^{n_{1}}\right)}\right), \operatorname{ad}\left(y^{\left(p^{n_{2}}\right)}\right)\right]$, of degree $\left(p^{n_{1}}-2, p^{n_{2}}-2\right)$;

(6) the degree derivation ad $h$, which has degree $(0,0)$ and acts as $(\operatorname{ad} h)\left(x^{(i)} y^{(j)}\right)=$ $(i+j-2) x^{(i)} y^{(j)}$.

Note that the derivations under (1) and (2) are powers of inner derivations of $H\left(2: \mathbf{n} ; \omega_{0}\right)$; together with the inner derivations, they span its $p$-closure $\overline{H\left(2: \mathbf{n} ; \omega_{0}\right)}$. We have denoted the derivations under (3), (4) and (5) as restrictions of inner derivations of $\tilde{H}\left(2: \mathbf{n} ; \omega_{0}\right)$. Finally, the degree derivation is the restriction of the inner derivation ad $h$ of $W(2: \mathbf{n})$, where the element $h=x \partial / \partial x+y \partial / \partial y$ has no analogue in the Poisson bracket notation which we have adopted. 
All derivations listed above except the degree derivation (because $p>3$, but see Remark 4.3 for $p=3$ ) are skew with respect to $\lambda$ (by direct verification, or from [29, Proposition 2.2]), and hence $\operatorname{dim}\left(H^{2}(L, \mathbb{F})\right)=\operatorname{dim}(\operatorname{Der}(L) / \operatorname{ad}(L))-1=n_{1}+n_{2}+1$ for $L$ and $p>3$ (see, for example, [29, Theorem. 2.4] and [26]). A set of $n_{1}+n_{2}+1$ cocycles of $L=H\left(2: \mathbf{n} ; \omega_{0}\right)$ which form a basis of $H^{2}(L, \mathbb{F})$ can be obtained from the skew derivations described above according to the procedure described earlier. Note that since $\lambda: L \otimes L \rightarrow \mathbb{F}$ is a graded map of degree $\left(-p^{n_{1}}+3,-p^{n_{2}}+3\right)$ (where the trivial module $\mathbb{F}$ is assigned degree zero), the cocycles thus obtained are homogeneous with respect to the grading of $Z^{2}(L, \mathbb{F})$ inherited by the $\mathbb{Z}^{2}$-grading of $L$. A conclusion which is more relevant for us is that the universal central extension $M$ of $L$ (see Remark 5.2) inherits a $\mathbb{Z}^{2}$-grading from $L$, and the central elements corresponding (see Remark 4.1) to the cocycles obtained are homogeneous. We record the degrees explicitly: if $D$ is a homogeneous skew derivation of degree $(i, j)$, then the central elements of the universal central extension $M$ of $L$ corresponding to the cocycle $\varphi(D)$ acquires degree $\left(p^{n_{1}}-3-i, p^{n_{2}}-3-j\right)$.

REMARK 4.1. Strictly speaking, central elements of $M$ correspond naturally to elements of the second homology group $\mathrm{H}_{2}(L, \mathbb{F})$, and not of its dual $H^{2}(L, \mathbb{F})$. There is, however, a natural correspondence between the homogeneous components in the $A$-gradings of $H_{2}(L, \mathbb{F})$ and its dual $H^{2}(L, \mathbb{F})$, reversing the sign of the degrees. The fact that all these components are one-dimensional in the present case (and in the case of $L=H\left(2: \mathbf{n} ; \omega_{2}\right)$ below $)$ justifies our abuse of language.

Now we turn our attention to the filtered algebra $H\left(2: \mathbf{n} ; \omega_{2}\right)$. This algebra has a non-degenerate associative form $\lambda$ (see [11, Theorem 7]), defined by the same formula given above for $H\left(2: \mathbf{n} ; \omega_{0}\right)$ with, in addition, $\lambda(\bar{x} \bar{y}, \bar{x} \bar{y})=1$ and $\lambda(\cdot, \cdot)=0$ in all remaining cases. Again from [7] (or the original source [41, Theorem 3.2]), all outer derivations of $H\left(2: \mathbf{n} ; \omega_{2}\right)$ are lifted from part of those of its associated graded algebra, which is $H\left(2: \mathbf{n} ; \omega_{0}\right) \oplus\langle\bar{x} \bar{y}\rangle$. Specifically, a basis for the space of outer derivations of $H\left(2: \mathbf{n} ; \omega_{2}\right)$ is given by

$$
(\operatorname{ad} x)^{p^{r}} \text { for } 0<r \leq n_{2} \quad \text { and } \quad(\operatorname{ad} y)^{p^{s}} \text { for } 0<s \leq n_{1} .
$$

(Note that $(\operatorname{ad} x)^{\rho^{n_{2}}}$ and $(\operatorname{ad} y)^{p^{n_{1}}}$ induce the derivations $\operatorname{ad}\left(x^{\left(p^{\left.n_{1}\right)}\right)}\right)$ and $\operatorname{ad}\left(y^{\left(p^{n_{2}}\right)}\right)$ on the associated graded algebra.) In particular, all derivations of $L=H\left(2: \mathbf{n} ; \omega_{2}\right)$ belong to its $p$-closure in $\operatorname{Der}(L)$ and, consequently, they are all skew.

So far our assumption that $p>3$ was in force. However, the $n_{1}+n_{2}$ derivations of $L=H\left(2: \mathbf{n} ; \omega_{2}\right)$ which we have described clearly remain linearly independent in $\operatorname{Der}(L) / \operatorname{ad}(L)$ also for smaller characteristics, provided in characteristic two we replace the degree derivation (which coincides with the inner derivation ad $(x \partial / \partial x-$ $y \partial / \partial y)=\operatorname{ad}(\mathscr{D}(x y))$ in that case) with $\operatorname{ad}(x \partial / \partial x)$. In Section 6, we will identify 
$H\left(2: \mathbf{n} ; \omega_{2}\right)$ with an algebra of Albert and Frank (a special type of Block algebra, see Section 3), and we will prove (extending results obtained in [11] for characteristic $p>3$ ) that its space of outer derivations has dimension $n_{1}+n_{2}$, in every positive characteristic. Consequently, the derivations of $H\left(2: \mathbf{n} ; \omega_{2}\right)$ described above, but with $\operatorname{ad}(x \partial / \partial x)$ replacing the degree derivation (a change which is only relevant in characteristic two), form a basis for its space of outer derivations regardless of the characteristic.

Setting $\varphi_{r}=\varphi\left((\operatorname{ad} x)^{p^{r}}\right)$ and $\psi_{s}=\varphi\left((\operatorname{ad} y)^{p^{s}}\right)$, the following result follows by direct computation.

THEOREM 4.2. A basis for the second cohomology group $H^{2}(L, \mathbb{F})$ of the Hamiltonian Lie algebra $L=H\left(2: \mathbf{n} ; \omega_{2}\right)$ over a field $\mathbb{F}$ of odd characteristic is given by the classes of the cocycles $\varphi_{r}$ and $\psi_{s}$, for $0<r \leq n_{2}$ and $0<s \leq n_{1}$, as defined by the following formulas:

$$
\begin{aligned}
& \varphi_{r}\left(x^{(i)} y^{(j)}, x^{(k)} y^{(l)}\right)=\left\{\begin{array}{lc}
(-1)^{i+j} & \text { if }(i+k, j+l) \equiv\left(0, p^{r}\right) \\
0 & \left(\bmod \left(p^{n_{1}}-1, p^{n_{2}}-1\right)\right)
\end{array}\right. \\
& \psi_{s}\left(x^{(i)} y^{(j)}, x^{(k)} y^{(l)}\right)=\left\{\begin{array}{cc}
(-1)^{i+j+1} & \text { if }(i+k, j+l) \equiv\left(p^{s}, 0\right) \\
0 & \left(\bmod \left(p^{n_{1}}-1, p^{n_{2}}-1\right)\right) \\
0 & \text { otherwise. }
\end{array}\right.
\end{aligned}
$$

The $A$-degrees of the cocycles $\varphi_{r}$ and $\psi_{s}$ are $\left(2,-p^{r}+2\right)$ and $\left(-p^{s}+2,2\right)$, respectively. The central elements of the universal central extension $M$ of $L=$ $H\left(2: \mathbf{n} ; \omega_{2}\right)$ (see Remark 5.2) corresponding to them acquire $A$-degree $\left(-2, p^{r}-2\right)$ and $\left(p^{s}-2,-2\right)$, respectively.

REMARK 4.3. It is easy to verify that the degree derivation of $H\left(2: \mathbf{n} ; \omega_{0}\right)$ acts on $\lambda$, considered as an element of $(L \otimes L)^{*}$, as multiplication by 6 . In particular, when the characteristic is three (or two, but see Remark 4.5 concerning this case) the degree derivation is skew (see, for example, [29, Proposition 2.2]), and the second cohomology group of $H\left(2: \mathbf{n} ; \omega_{0}\right)$ becomes larger. Also, the derivation algebra of $L$ can be larger (see [58, page 197]). In particular, the graded Hamiltonian algebra $L=$ $H\left(2:(1,1) ; \omega_{0}\right)$ in characteristic three is a classical Lie algebra of type $A_{2}$, namely, $L$ is isomorphic to the quotient of $\mathrm{sl}_{3}$ modulo its one-dimensional center, see [53, Lemma 6.4]. According to [11, Corollary 3] and the identification of Hamiltonian algebras with Block algebras, this is the only instance for $p>2$ where an algebra $H\left(2: \mathbf{n} ; \omega_{0}\right)$ or $H\left(2: \mathbf{n} ; \omega_{2}\right)$ is isomorphic with a classical algebra. It is well known that $\operatorname{Der}(L)$ is a fourteen-dimensional classical algebra of type $G_{2}$ (cf. [53, page 678]), hence $\operatorname{dim}(\operatorname{Der}(L) / \operatorname{ad}(L))=7$. The additional three derivations with respect to those 
described earlier can be obtained from ad $x^{(3)}$, ad $y^{(3)}$ and ad $\bar{x} \bar{y}$ by conjugation under the automorphism $x^{(i)} y^{(j)} \mapsto(-1)^{i j} x^{(2-i)} y^{(2-j)}$ of $L$. Since all derivations are skew in this case, we conclude that $H^{2}(L, \mathbb{F}$ ) has dimension 7 (but see [59, page 38] for another proof of this fact).

It is easily checked that exactly one of those three 'exceptional derivations' of $H\left(2:(1,1) ; \omega_{0}\right)$ extends to a derivation of $H\left(2:\left(1, n_{2}\right) ; \omega_{0}\right)$ for $n_{2}>1$. These additional derivations account for all the exceptions in characteristic three with respect to the description of outer derivations of $H\left(2: \mathbf{n} ; \omega_{0}\right)$ in higher characteristic given earlier in this section. This follows from [53, Proposition 4.3] and a general fact about derivations of nonnegative degree (in the standard grading) of graded Lie algebras of Cartan type, see [58, Chapter 4, Proposition 8.3]. The derivations of $L=H\left(2: \mathbf{n} ; \omega_{0}\right)$ in characteristic three can be summarized as follows: $\operatorname{dim}(\operatorname{Der}(L) / \operatorname{ad}(L))$ equals $n_{1}+n_{2}+2$ if $n_{1}, n_{2}>1$ (like in higher characteristic), it equals $n_{1}+n_{2}+3$ if $n_{1}=1<n_{2}$, and it equals 7 if $n_{1}=n_{2}=1$. Since all derivations of $L$ are skew in characteristic three, we have $\operatorname{dim}\left(H^{2}(L, \mathbb{F})\right)=\operatorname{dim}(\operatorname{Der}(L) / \operatorname{ad}(L))$.

RemarK 4.4. Again according to [53, Proposition 4.3] and [58, Chapter 4, Proposition 8.3], the derivations of a simple algebra $H\left(2: \mathbf{n} ; \omega_{0}\right)$ in characteristic two (hence with $n_{1}, n_{2}>1$ ) allow the same description as in characteristic greater than three, by the list given earlier in this section, except that the degree derivation in item (6) (which is inner in characteristic two, as it coincides with ad $x y$ ) should be replaced with the derivation acting as $D\left(x^{(i)} y^{(j)}\right)=(i-1) x^{(i)} y^{(j)}$. In particular, $\operatorname{Der}(L) / \operatorname{ad}(L)$ has dimension $n_{1}+n_{2}+2$.

The algebra $H\left(2:\left(1, n_{2}\right) ; \omega_{0}\right)$ in characteristic two has more derivations than usual. Since it is a semidirect product of a simple Zassenhaus algebra by its adjoint module, its derivations can be easily calculated from those of the Zassenhaus algebra (see Remark 4.6). In fact, if $L$ is a semidirect product of a simple algebra $S$ by its adjoint module, then $\operatorname{dim}(\operatorname{Der}(L))=2 \operatorname{dim}(\operatorname{Der}(S))+2$ in characteristic two, and $\operatorname{dim}(\operatorname{Der}(L))=2 \operatorname{dim}(\operatorname{Der}(S))+1$ otherwise. Consequently, $\operatorname{Der}(L) / \operatorname{ad}(L)$ has dimension $2 n_{2}+2$ for $L=H\left(2:\left(1, n_{2}\right) ; \omega_{0}\right)$. Alternatively, since $H\left(2:\left(1, n_{2}\right) ; \omega_{0}\right)$ is the tensor product of a simple Zassenhaus algebra with a ring of divided powers $\mathbb{F}[z: 1]$, the conclusion follows from [12, Theorem 7.1].

REMARK 4.5. In characteristic two the argument which relates derivations of $L$ into $L^{*}$ and the second cohomology group of $L$ needs to be modified as follows.

The image of the map $Z^{2}(L, \mathbb{F}) \rightarrow Z^{1}\left(L, L^{*}\right)$ consists of all derivations which are alternating, in the sense that $D(\xi)(\xi)=0$ for all $\xi \in L$. This condition is equivalent to being skew in odd characteristic, but is stronger in characteristic two. (It is convenient to reserve the term skew for the weaker condition, as it applies in slightly greater generality; see [29], where Lemma 1.1 remains valid for skew derivations, but 
not for alternating derivations, in characteristic two.) In presence of a nondegenerate associative form $\lambda$, a derivation $D$ of $L$ will be called alternating with respect to $\lambda$ if $\lambda(D(\xi), \xi)=0$ for all $\xi \in L$. Since inner derivations of $L$ are alternating, there is an isomorphism of $H^{2}(L, \mathbb{F})$ with the quotient of the space of alternating derivations by the space of inner derivations of $L$. Note that while the space of skew derivations is a $p$-subalgebra of $\operatorname{Der}(L)$ (in every characteristic), the space of alternating derivations is a Lie subalgebra but need not be a $p$-subalgebra in characteristic two (as shown by the examples below).

In order to verify that a skew derivation is alternating it suffices to check that $D(\xi)(\xi)=0$ for all elements $\xi$ of some basis of $L$. Also, if $L$ is graded and its associative form is homogeneous with respect to the grading (as is the case for the $\mathbb{Z}^{2}$-grading of $H\left(2: \mathbf{n} ; \omega_{0}\right)$ and the $A$-grading of $\left.H\left(2: \mathbf{n} ; \omega_{2}\right)\right)$ it is enough to check derivations which are homogeneous with respect to the grading, because the alternating derivations form a graded subalgebra (as well as the skew derivations).

For $L=H\left(2: \mathbf{n} ; \omega_{0}\right)$ with $n_{1}, n_{2}>1$, all derivations described earlier in this section (taking Remark 4.4 into account) are alternating, except ad $x^{\left(p^{\left.n_{1}\right)}\right.}$ and ad $y^{\left(p^{n_{2}}\right)}$, which are only skew. Therefore, $H^{2}(L, \mathbb{F})$ has dimension $n_{1}+n_{2}$. An examination of $L=H\left(2:\left(1, n_{2}\right) ; \omega_{0}\right)$ shows that the alternating derivations are exactly those which normalize the Zassenhaus subalgebra $\left\langle x y^{(j)}: j=0, \ldots, 2^{n_{2}}-2\right\rangle$ (together with the inner derivations). These correspond to the derivations described under items (1), (5) and (6) in the list given earlier in this section, and we conclude that $H^{2}(L, \mathbb{F})$ has dimension $n_{1}+n_{2}$ in this case, too.

In the case of $L=H\left(2: \mathbf{n} ; \omega_{2}\right)$ all alternating derivations are inner. As a consequence, in characteristic two we have $H^{2}(L, \mathbb{F})=0$.

REMARK 4.6. Recalling from Section 3 that the simple Zassenhaus algebra $L=$ $W(1: n)^{(1)}$ in characteristic two is isomorphic with $L=H\left(2:(1, n-1) ; \omega_{2}\right)$, the previous remark shows that its second cohomology group $H^{2}(L, \mathbb{F})$ vanishes. By contrast, the second cohomology group of the Zassenhaus algebra $W(1: n)$ in odd characteristic has dimension one if $p>3$, and dimension $n-1$ if $p=3$ (as a special case of [30, Theorem. 3.2] or [26]). The second cohomology group of the simple Zassenhaus algebra was also computed in [27, Theorem 2]; however, note that the central extensions of $W(1: n)^{(1)}$ in characteristic two which are exhibited there are not Lie algebras in the common sense, because their multiplication is (skew-)symmetric but not alternating. For the sake of completeness we mention that the algebra of outer derivations of the simple Zassenhaus algebra $W(1: n)^{(1)}$ has dimension $n-1$ if $p$ is odd and $n$ if $p=2$. This is well known, but the case where $p=2$ is also a consequence of Theorem 6.2.

REMARK 4.7. The last sentence in [11] claims that there is no isomorphism between 
a Block algebra and an exceptional (classical) simple algebra except when $p=2$ and the algebra has dimension 14, since otherwise their dimensions are distinct. This may leave some doubt on whether in characteristic two $H\left(2:(2,2) ; \omega_{0}\right)$ might be isomorphic with a simple algebra of type $G_{2}$ (which, in turn, is isomorphic with the quotient of $\mathrm{sl}_{4}$ by its one-dimensional center). However, according to [59] the second cohomology group of the latter has dimension 7, and this fact together with Remark 4.5 excludes the possibility of an isomorphism.

\section{Some cyclic gradings of $H\left(2: n ; \omega_{2}\right)$}

The $A$-grading of $H\left(2: \mathbf{n} ; \omega_{2}\right)$ defined in Section 3 leads in a natural way to several gradings (here called specializations) over cyclic quotients $\bar{A}$ of $A=$ $\mathbb{Z}^{2} /\left\langle\left(p^{n_{1}}-1, p^{n_{2}}-1\right)\right\rangle$. More precisely, for any pair of integers $(R, S)$ and any divisor $N$ of $R\left(p^{n_{1}}-1\right)+S\left(p^{n_{2}}-1\right)$ we have a group homomorphism $\mu: A \rightarrow$ $\bar{A}=\mathbb{Z} / N \mathbb{Z}$ given by $\mu(i, j)=R i+S j+N \mathbb{Z}$. Correspondingly, we obtain an $\bar{A}$ grading $L=\bigoplus_{k \in \bar{A}} L_{k}$ by setting $L_{k}=\sum_{\mu(i, j)=k} L_{i, j}$. In what follows we set $N=\left|R\left(p^{n_{1}}-1\right)+S\left(p^{n_{2}}-1\right)\right|$, since the remaining cases can be obtained from these gradings through a further specialization. Also, it is no loss to assume that the homomorphism is surjective, which amounts to choosing $R$ and $S$ relatively prime (because of our choice of $N$ ). To help visualizing the grading thus obtained, it may be convenient to arrange the monomials in $H\left(2: \mathbf{n} ; \omega_{2}\right)$ in a $\left(p^{n_{1}} \times p^{n_{2}}\right)$-array according to the degrees of $x$ and $y$, and think of the specialization process as slicing the $A$-grading according to some specified direction.

The simplest specialization where $(R, S)=(0,-1)$ will be useful in Section 7 . This is a $\mathbb{Z} / N \mathbb{Z}$-grading with $N=p^{n_{2}}-1$, every component has dimension $p^{n_{1}}$ and is spanned by all monomials where $y$ has a given degree $1 \leq j \leq p^{n_{2}}-2$, except the component of degree 1 , which has dimension $2 p^{n_{1}}-1$ and is spanned by all monomials where $y$ has degree 0 or $p^{n_{2}}-1$. The component of degree 0 is isomorphic with a Zassenhaus algebra $W\left(1: n_{1}\right)$.

The following two specializations of the $A$-grading of $H\left(2: \mathbf{n} ; \omega_{2}\right)$ are more interesting.

5.1. A grading related to graded Lie algebras of maximal class Let $(R, S)=$ $\left(-p^{n_{2}},-1\right)$. Then $N=p^{n}-1$, and all components $L_{k}$ are one-dimensional. In fact, $L_{i p^{n_{2}}+j}=\left\langle x^{\left(p^{\left.n_{1}-i\right)}\right.} y^{\left(p^{\left.n_{2}-j\right)}\right\rangle}\right\rangle$, for $0<i \leq p^{n_{1}}$ and $0<j \leq p^{n_{2}}$ with $(i, j) \neq\left(p^{n_{1}}, p^{n_{2}}\right)$. Furthermore, $L$ has an outer derivation

$$
D=\bar{y} \frac{\partial}{\partial x}+(1+e) \frac{\partial}{\partial y}
$$


which is non-singular and homogeneous of degree one (with respect to the grading under consideration). Hence $D$ permutes the components of the grading cyclically, namely $D L_{k}=L_{k+1}$ for all $i$. (Recall from Section 3 that we write $D$ for ad $D$, since the latter acts the same way as a Lie algebra derivation of $P\left(2: \mathbf{n} ; \omega_{2}\right)$ as $D$ acts as a derivation of the associative structure.) In fact, we have

$$
D x^{(i)} y^{(j)}=\bar{y} x^{(i-1)} y^{(j)}+(1+e) x^{(i)} y^{(j-1)}= \begin{cases}x^{(i-1)} \bar{y} & \text { if } j=0 \\ (1+e) x^{(i)} y^{(j-1)} & \text { if } j>0\end{cases}
$$

In particular, the derivation $D$ is periodic of period $p^{n}-1$. Note that $D$ is the derivation denoted by $D_{2}$ in $[7$, p. 911$]$, viewing the Hamiltonian algebra $H\left(2: \mathbf{n} ; \omega_{2}\right)$ as the special algebra $S\left(2: \mathbf{n} ; \omega_{2}\right)$.

We quote from [37] the following definition.

DEFINITION 5.1. We say that a finite-dimensional Lie algebra $L$ admits a nonsingular derivation $D$ agreeing with $a \mathbb{Z} / N \mathbb{Z}$-grading $L=\bigoplus_{k \in \mathbb{Z} / N \mathbb{Z}} L_{k}$ if $D L_{k}=L_{k+1}$ for all $k \in \mathbb{Z} / N \mathbb{Z}$.

It will be convenient to allow any finite cyclic group to replace $\mathbb{Z} / N \mathbb{Z}$ in the definition, provided we specify a distinguished generator of it (to play the role of 1). The situation described in Definition 5.1 where all components $L_{k}$ have dimension one played a crucial role in [52] and [51].

Suppose $L$ is a finite-dimensional Lie algebra possessing a nonsingular derivation which agrees with a $\mathbb{Z} / N \mathbb{Z}$-grading with one-dimensional components. We build the corresponding twisted loop algebra $\bigoplus_{k \in \mathbb{Z}} L_{\bar{k}} \otimes t^{k}$ inside $L \otimes_{\mathbb{F}} \mathbb{F}\left[t, t^{-1}\right]$, where $\bar{k}$ denotes the residue class of $k$ modulo $N$. The Lie algebra spanned by its positive part $\bigoplus_{k>0} L_{\bar{k}} \otimes t^{k}$ together with its derivation $D \otimes t$ is a graded Lie algebra of maximal class in the sense of [22]. With a harmless abuse of language we will call the latter the loop algebra of $L$.

In particular, the loop algebra of $H\left(2: \mathbf{n} ; \omega_{2}\right)$ with respect to the derivation $D$ and the grading which we have just constructed is a graded Lie algebra of maximal class, and precisely one of those which we have named after Albert-Frank-Shalev in [22]. We will come back to this grading in Section 6.

REMARK 5.2. We comment briefly on the relevance of the second cohomology group of $H\left(2: \mathbf{n} ; \omega_{2}\right)$, which we have discussed in Section 4 , to presentations of the algebras of Albert-Frank-Shalev $A F S(a, b, n, p)$ (see Section 6 for their definition). Although these algebras are not finitely presented, it is proved in [25] that they are quotients of certain finitely presented Lie algebras modulo their second centers. Knowledge of the second cohomology group of $H\left(2: \mathbf{n} ; \omega_{2}\right)$ sheds light on these particular extensions of the algebras of Albert-Frank-Shalev, as we illustrate below. 
The second cohomology group of $H\left(2: \mathbf{n} ; \omega_{0}\right)$ plays a similar role in [3] and [21, Section 4].

Recall that every perfect Lie algebra $L$ has a universal central extension $0 \rightarrow Z \rightarrow$ $M \rightarrow L \rightarrow 0$ (see [59, Section 1]). In particular, $M / Z \cong L$, so we may view $L$ as a quotient of $M$, and $Z \cong H_{2}(L, \mathbb{F}) \cong H^{2}(L, \mathbb{F})^{*}$ as vector spaces. According to [10, Theorem 2.2], every derivation of $L$ lifts to a derivation of $M$; if $L$ is centerless, the lift is unique, therefore $\operatorname{Der}(M) \cong \operatorname{Der}(L)$, and $M$ is a $\operatorname{Der}(L)$-module in a natural way. In the case of $L=H\left(2: \mathbf{n} ; \omega_{2}\right)$, we claim that $\operatorname{Der}(L)$ acts trivially on $Z$, which coincides with the center $\zeta(M)$ of $M$ here. In fact, for a perfect and centerless Lie algebra $L$, the dual module $\zeta(M)^{*}$ and $H^{2}(L, \mathbb{F})$ are easily seen to be isomorphic not only as vector spaces, but as $\operatorname{Der}(L)$-modules. Now assume, in addition, that the characteristic is odd and that $L$ has a nonsingular associative form $\lambda$. Then the isomorphism of $H^{2}(L, \mathbb{F})$ with $\operatorname{SkDer}(L) / \operatorname{ad}(L)$ described in Section 4 is also an isomorphism of $\operatorname{Der}(L)$ modules (with respect to the adjoint action of $\operatorname{Der}(L)$ on itself). Since $\operatorname{Der}(L) / \operatorname{ad}(L)$ is abelian for $L=H\left(2: \mathbf{n} ; \omega_{2}\right)$, our claim follows. Therefore, the center of the extension of $M$ by $\langle D\rangle$, where $D$ is the nonsingular derivation (5.1), coincides with $\zeta(M)$ and, in particular, is nonzero according to Theorem 4.2, in odd characteristic. It follows that the loop algebra $\tilde{M}$ of $M$ with respect to $D$ has an infinite-dimensional center. The quotient of $\tilde{M}$ by its center is isomorphic with $\tilde{L}$. A standard result of B. H. Neumann recalled in [20] as Theorem 6 implies that the quotient of a finitely generated Lie algebra modulo an infinite-dimensional central ideal cannot be finitely presented; in particular, $\tilde{L} \cong \tilde{M} / \zeta(\tilde{M})$ is not finitely presented.

The main result of [25] shows that a suitable central extension of $\tilde{M}$ is finitely presented. (The need to take a further central extension to obtain a finitely presented algebra is due to the fact that the second cohomology group of a loop algebra, besides depending on the second cohomology group of the underlying finite-dimensional algebra, includes a component arising from associative forms of the latter and the cyclic homology of the polynomial ring $\mathbb{E}[t]$ which we are tensoring with. We will not discuss this point further here, but see [62].) According to our identification in Theorem 6.1 of the algebras of Albert and Frank with Hamiltonian algebras $H\left(2: \mathbf{n} ; \omega_{2}\right)$, the second cohomology group of the latter discussed in Theorem 4.2 can be recognized in (part of) the central elements of the finitely presented central extensions of the Albert-Frank-Shalev algebras considered in [25]. More precisely, the $\bar{A}$-grading of $L=H\left(2: \mathbf{n} ; \omega_{2}\right)$ (like any other grading) extends uniquely to a grading of its universal central extension $M$. The central elements of the latter corresponding (recall Remark 4.1) to the cocycles $\varphi_{r}$ and $\psi_{s}$ of Theorem 4.2 (in odd characteristic) occur in degrees $2 q-p^{r}+2$ and $2 q-q p^{s}+2$, respectively, and give rise to central elements of $\tilde{M}$, the loop algebra of $M$ with respect to $D$, in all degrees congruent to these modulo $\operatorname{dim}(L)=p^{n_{1}+n_{2}}-1$. These central elements can be recognized in the list given in [25, pages 399-400], in the special case of the algebra $A F S(a, n, n, p)$. 
(Additional complications arise in the case of characteristic two, which we have neglected here for simplicity.)

By symmetry, the case $(R, S)=\left(-1,-p^{n_{1}}\right)$ is completely analogous. In this case

$$
D^{p^{n_{2}}}=(1+e) \frac{\partial}{\partial x}+\bar{x} \frac{\partial}{\partial y}
$$

is a non-singular derivation which permutes the components cyclically. This latter grading is just one instance of a whole set of $\bar{A}$-gradings which can be obtained from the former $\bar{A}$-grading by an application of an automorphism of the grading group $\bar{A}$. More precisely, $L$ is also graded by the subspaces $\tilde{L}_{i}=L_{k i}$, where $k$ is any integer with $\left(k, p^{n}-1\right)=1$. In general, a derivation $\tilde{D}$ such that $\tilde{D} \tilde{L}_{i}=\tilde{L}_{i+1}$ need not exist. However, it certainly does if $k$ is a power of $p$, because then $D^{p^{\prime}} \tilde{L}_{i}=\tilde{L}_{i+1}$, if $\tilde{L}_{i}=L_{p^{\prime} i}$. This way of obtaining new gradings is related to the process of deflation for graded Lie algebras of maximal class introduced in [22]. In fact, the loop algebra of $L$ with respect to its grading given by the subspaces $\tilde{L}_{i}=L_{p i}$ and its derivation $D^{p}$ is the deflation of the loop algebra of $L$ with respect to its grading given by the subspaces $L_{i}$ and its derivation $D$.

5.2. A thin grading Let $(R, S)=\left(-p^{n_{2}}+1,-1\right)$. This is a $\mathbb{Z} / N \mathbb{Z}$-grading with $N=p^{n_{1}}\left(p^{n_{2}}-1\right)$, and the components have dimension one or two. In the present grading, the two-dimensional components are those of degree $i\left(p^{n_{2}}-1\right)+1$ for $1<i \leq p^{n_{1}}$. In particular, $L_{1}=\langle x, \bar{y}\rangle$. This grading fits the following definition.

DEFINITION 5.3. A grading $L=\bigoplus_{k \in \mathbb{Z} / N \mathbb{Z}} L_{k}$ of a (finite-dimensional) Lie algebra $L$ over a field $\mathbb{F}$ is called thin if $L_{1}$ is two-dimensional, and the following covering property holds

for all $k \in \mathbb{Z} / N \mathbb{Z}$, and all $u \in L_{k}, u \neq 0$, we have $L_{k+1}=\left[u, L_{1}\right]$.

Again, the definition is motivated by an analogous one for positively graded, possibly infinite-dimensional Lie algebras. In fact, given a thin $\mathbb{Z} / N \mathbb{Z}$-grading of a finite-dimensional Lie algebra $L$, the (positive part of the twisted) loop algebra $\bigoplus_{k>0} L_{\bar{k}} \otimes \mathbb{F} t^{k}$ is a thin Lie algebra in the sense of the following definition (see [20] for background).

DEFINITION 5.4. A graded Lie algebra $L=\bigoplus_{k=1}^{\infty} L_{k}$ is called thin if $L_{1}$ is twodimensional, and the following covering property holds

for all $k \geq 1$, and all $u \in L_{k}, u \neq 0$, we have $L_{k+1}=\left[u, L_{1}\right]$. 
With both definitions, it follows from the covering property that $L$ is generated by $L_{1}$, and that $\operatorname{dim}\left(L_{k}\right) \leq 2$ for all $k$. We call a homogeneous component $L_{k}$ of dimension 2 a diamond. The diamonds will be numbered in the natural order of occurrence (cyclic starting from $L_{1}$ in case of Definition 5.3). Therefore, $L_{1}$ is the first diamond. If there are no other diamonds in case of Definition 5.4, then $L$ is an algebra of maximal class (see [22]). We refer to the finite sequence of one-dimensional homogeneous components between two consecutive diamonds as a chain.

We must point out that there are also instances where a thin Lie algebra in the sense of Definition 5.4 is constructed as a loop algebra from a suitable grading of a finite dimensional simple (Hamiltonian) Lie algebra which does not quite fit Definition 5.3, but requires the intervention of a nonsingular outer derivation, very much like in the construction of Lie algebras of maximal class described in the previous subsection (see $[3,2])$. These algebras are not needed in this paper, however.

The fact that an algebra with a thin grading in the sense of Definition 5.3 gives rise to a thin Lie algebra in the sense of Definition 5.4 via the loop algebra construction allows one to apply to the former setting arguments and results originally formulated for the latter. For example, results from [23], extended in [4], imply that in a finitedimensional Lie algebra $L$ over a field of arbitrary characteristic with a thin grading, the second diamond can only occur in degree $3,5, q$, or $2 q-1$, where $q$ is a power of the characteristic. Some care is needed in carrying definitions over from the infinitedimensional setting to the present one, where the degree of a homogeneous element is an integer defined only modulo the order $N$ of the grading group: when speaking of the degree where the second diamond occurs, we actually refer to the smallest integer greater than one in which degree a diamond occurs.

Suppose now that $L$ is a finite-dimensional Lie algebra with a thin grading, and suppose that the second diamond occurs in degree $2 q-1$, where $q$ is a power of the characteristic. According to [19], we have

$$
C_{L_{1}}\left(L_{2}\right)=C_{L_{1}}\left(L_{3}\right)=\cdots=C_{L_{1}}\left(L_{2 q-3}\right)=\langle Y\rangle,
$$

provided the characteristic is odd; this fails in characteristic two, as is shown in [31,34]. We let $X \in L_{1} \backslash\langle Y\rangle$, so that $X$ and $Y$ generate $L$. (Note that with respect to the analogous situation in [20] we have switched to capital letters for the generators $X$ and $Y$, to avoid conflict of meaning with the variables we use for divided powers.)

Suppose that $L_{k}$ is any diamond of $L$, and let $L_{k-1}=\langle V\rangle$. It is not difficult to show, as in [20], that $[V, X, Y]+[V, Y, X]=[V, Y, Y]=0$, and to deduce that

$$
[V, Y, X]=\lambda[V, X, X]
$$

for some $\lambda \in \mathbb{F} \cup\{\infty\}$, to be read as $[V, X, X]=0$ when $\lambda=\infty$. As in the infinite-dimensional setting of [20] we will say that the diamond in degree $k$ has 
type $\lambda$. Note that $\lambda$ depends on the choice made for the generators $X$ and $Y$, but the type being finite, or infinite, does not. Strictly speaking, $\lambda=0$ cannot occur here, because the covering property would imply that $[V, Y]=0$ and so $L_{k}=\langle[V X]\rangle$, contradicting the assumption that $L_{k}$ is a diamond. However, there are situations (here and in other papers, like [20], [3] and [21]) where we have found natural and convenient to informally call fake diamonds certain one-dimensional components $L_{k}$, which thus may be assigned type 0 . This usually happens when the diamonds of some algebra with a thin grading (or some infinite-dimensional thin algebra) occur at regular intervals provided we include some fake diamonds. Unfortunately, which cocycle of the algebra gives rise to the central element in a fake diamond depends on the grading under consideration, and appears not to admit an intrinsic characterization, in terms of the algebra alone.

The $\mathbb{Z} / N \mathbb{Z}$-grading of $H\left(2: \mathbf{n} ; \omega_{2}\right)$ under consideration in this subsection is a thin grading, with diamonds in all degrees congruent to 1 modulo $q-1$, with the exception of degree $q$, where we have set $q=p^{n_{2}}$. In fact, here we may take $Y=\bar{y}$ and $X=x$, and the following computations show both the validity of the covering property and the location of the diamonds:

$$
\begin{aligned}
& \left\{x^{(i)} y^{(j)}, x\right\}=(1+e) x^{(i)} y^{(j-1)}, \\
& \left\{x^{(i)} y^{(j)}, \bar{y}\right\}=-x^{(i-1)} y^{(j)} y^{\left(p^{n_{2}}-2\right)}= \begin{cases}-x^{(i-1)} y^{\left(p^{n_{2}}-2\right)} & \text { if } j=0, \\
x^{(i-1)} \bar{y} & \text { if } j=1, \\
0 & \text { otherwise. }\end{cases}
\end{aligned}
$$

Taking $V=x^{(i)} y$, for $0<i<p^{n_{1}}$, we see that all diamonds have type $\infty$. Note that the elements $x^{(i)} y$ with $0 \leq i<p^{n_{1}}$, that is, the elements just above (in the sense of immediately preceding) the diamonds, if we include a fake diamond $L_{p^{n_{2}}}=\langle y\rangle$, span a subalgebra of $H\left(2: \mathbf{n} ; \omega_{2}\right)$ isomorphic with a Zassenhaus algebra $W\left(1: n_{1}\right)$. This feature will reappear in the different grading which we will consider in Section 7.

According to [20], the loop algebra of $H\left(2: \mathbf{n} ; \omega_{2}\right)$ with respect to this grading is the only thin maximal subalgebra of the algebra of maximal class $\operatorname{AFS}\left(0, n_{2}, n, p\right)$, which is the loop algebra of $H\left(2: \mathbf{n} ; \omega_{2}\right)$ with respect to the grading seen in the previous subsection. The grading of $H\left(2: \mathbf{n} ; \omega_{2}\right)$ extends uniquely to a grading of its universal central extension. The central elements of the latter corresponding to the cocycles $\varphi_{r}$ and $\psi_{s}$ of Theorem 4.2 (in odd characteristic) occur in degrees $2 q-p^{r}$ and $2 q-p^{s}(q-1)$, respectively.

\section{The Hamiltonian algebra $H\left(2: n ; \omega_{2}\right)$ as a Block algebra}

Benkart, Kostrikin and Kuznetsov proved in [7, Theorem 4.9] (using the classification of the modular simple Lie algebras of characteristic $p>7$ completed in [56]) 
that the only simple Lie algebras over an algebraically closed field of characteristic $p>7$ which admit a nonsingular derivation agreeing with a $\mathbb{Z} / N \mathbb{Z}$-grading with onedimensional components (in the terminology introduced in Definition 5.1) are of type $H\left(m: \mathbf{n} ; \omega_{2}\right)$. (In [7, Theorem 4.9] $N$ had the form $p^{n}-1$, but this was immaterial.) Later it was proved in [39] that $m$ necessarily equals two.

Simple Lie algebras with this property were considered earlier by Shalev in [50], who noted that certain Lie algebras introduced by Albert and Frank in [1] can be defined over the prime field $\mathbb{F}_{p}$ and enjoy the property. Shalev used them to build the first examples of insoluble graded Lie algebras of maximal class, as loop algebras of the algebras of Albert and Frank. The algebras of Albert and Frank and their loop algebras have been further discussed in [22], to which we conform our notation, as starting points for the construction of more graded Lie algebras of maximal class. A byproduct of the classification of graded Lie algebras of maximal class achieved in [24] and [33] (for odd and even characteristic, respectively) is a proof, independent of the classification of modular simple Lie algebras, that the only finite-dimensional Lie algebras which admit a nonsingular derivation agreeing with a $\mathbb{Z} / N \mathbb{Z}$-grading with one-dimensional components, where $N$ is any integer prime to the characteristic, are the algebras of Albert and Frank. If we drop the condition on $N$ but assume the simplicity of the algebra the result remains true in odd characteristic (extending the cited result of [7]), while in characteristic two there is exactly one further class of algebras joining the algebras of Albert and Frank and consists of the Lie algebras constructed in [32] and named Bi-Zassenhaus algebras (for which $N$ is two less than a power of two). Note that in characteristic two the simple Zassenhaus algebra of dimension $2^{n}-1$ has this property, the nonsingular derivation being given by $\operatorname{ad}\left(e_{0}\right)=\operatorname{ad}\left(E_{-1}+E_{2^{n}-2}\right)$ in the notation introduced in Section 2. In fact, with respect to its basis $\left\{e_{\alpha}\right\}$ the simple Zassenhaus algebra coincides with the algebra of Albert and Frank $A F(0,1, n, 2)$ defined below. (As we have mentioned in Section 3, it also coincides with $H\left(2: \mathbf{n} ; \omega_{2}\right)$.)

The above results indirectly imply that for $p>7$ the class of algebras of Albert and Frank considered by Shalev coincides with the class of Hamiltonian algebras $H\left(2: \mathbf{n} ; \omega_{2}\right)$. In fact, since the algebras of Albert and Frank are Block algebras this result holds for $p>3$ according to [13, Lemma 1.8.3] together with the remarks on the characteristic which we have made at the end of Section 3. In Theorem 6.1 we use the cyclic grading and the nonsingular derivation of the algebras of Albert and Frank employed by Shalev to find an explicit isomorphism of the latter with Hamiltonian algebras $H\left(2: \mathbf{n} ; \omega_{2}\right)$, in arbitrary (positive) characteristic. We compute the derivations of the algebras of Albert and Frank in Theorem 6.2, extending to arbitrary characteristic a result obtained by Block in [11] for $p>3$; in view of Theorem 6.1, the description of derivations of algebras $H\left(2: \mathbf{n} ; \omega_{2}\right)$ quoted in Section 4 for $p>3$ is also extended to arbitrary characteristic. 
For integers $0 \leq a<b<n$ (the choice made in [22], or for the equivalent choice $0<a<b \leq n$ made later in [24]), the algebra of Albert and Frank $A F(a, b, n, p)$ is the Lie algebra over $\mathbb{F}_{p^{n}}$ with basis $\left\{e_{\xi} \mid \xi \in \mathbb{F}_{p^{n}}^{*}\right\}$ and multiplication given by

$$
\left[e_{\xi}, e_{\eta}\right]=\left(\xi^{p^{a}} \eta^{p^{b}}-\xi^{p^{b}} \eta^{p^{a}}\right) e_{\xi+\eta}
$$

(where the right-hand side is interpreted as zero when $\xi+\eta=0$, for example by setting $e_{0}=0$ ). Thus, an algebra of Albert and Frank is a special instance of a Block algebra (see Section 3). It was shown in [50] that the algebra of Albert and Frank $S=A F(a, b, n, p)$ is defined over the prime field $\mathbb{F}_{p}$. Moreover, it has a graded basis $u_{0}, \ldots, u_{p^{n}-2}$ over $\mathbb{F}_{p}$, and a derivation $D$ such that $D u_{i}=u_{i+1}$ for all $i$, to be read modulo $p^{n}-1$. Explicitly, this basis is related to the original basis by the formulas $u_{i}=\sum_{\xi \in \mathbb{F}, n} \xi^{i-p^{a}-p^{b}} e_{\xi}$. Part of the multiplication table with respect to the new basis, namely, a description of the adjoint action of the element $u_{p^{a}+p^{b}}$, was computed in [50, Proposition 2.4] (but see also [22]). If we include the derivation $D$ into consideration we obtain the following statement: the split extension of $S$ by $\mathbb{F}_{p} D$ has a finite presentation on the generators $u_{0}, \ldots, u_{p^{n}-2}$ (with subscripts viewed modulo $p^{n}-1$ ) and $D$, with relations

$$
\left\{\begin{array}{l}
D u_{i}=u_{i+1} \\
{\left[u_{p^{a}+p^{b}}, u_{p^{a}}\right]=-u_{2 p^{a}+p^{b}},} \\
{\left[u_{p^{a}+p^{b}}, u_{p^{b}}\right]=u_{p^{a}+2 p^{b}},} \\
{\left[u_{p^{a}+p^{b}}, u_{j}\right]=0 \quad \text { otherwise. }}
\end{array}\right.
$$

This implies at once that $S$ is defined over the prime field. Note that the above formulas differ in sign from those given in [22, page 4028], which were incorrectly quoted from [50, Proposition 2.4]. That mistake amounts to using the algebra with the opposite multiplication, and caused no serious consequence in [22]. Note also that here we have written the derivation $D$ on the left, differently from [22], and hence $\left[u_{i}, D\right]=-\left[D, u_{i}\right]=-D u_{i}$.

In the terminology introduced in Definition 5.1, the nonsingular derivation $D$ agrees with the $\mathbb{Z} / N \mathbb{Z}$-grading $S=\bigoplus_{k \in \mathbb{Z} / N \mathbb{Z}} S_{k}$, where $N=p^{n}-1$ and $S_{k}=\left\langle u_{k}\right\rangle$. We will take advantage of the similar grading of $H\left(2: \mathbf{n} ; \omega_{2}\right)$ defined in Subsection 5.1 to construct an isomorphism between $S=A F(a, b, n, p)$ and $H\left(2: \mathbf{n} ; \omega_{2}\right)$, where $\mathbf{n}=$ $(n-b+a, b-a)$. Since the map $e_{\xi} \mapsto e_{\xi p^{a}}$ gives an isomorphism of $A F(a, b, n, p)$ with $A F(0, b-a, n, p)$ we may restrict ourselves to the case $a=0$. We will construct a Lie algebra isomorphism $\sigma$ from the extension of $S$ by $\langle D\rangle$ to the extension of $L$ by $\langle D\rangle$, where $D=\bar{y} \partial / \partial x+(1+e) \partial / \partial y$. (Using the same symbol for the derivation $D$ of both algebras should create no confusion.) If we set

$$
\sigma\left(u_{i p^{n_{2}}+j}\right)=-x^{\left(p^{n_{1}}-i\right)} y^{\left(p^{n_{2}}-j\right)}
$$


for $0<i \leq p^{n_{1}}$ and $0<j \leq p^{n_{2}}$ with $(i, j) \neq\left(p^{n_{1}}, p^{n_{2}}\right)$, we clearly have $D\left(\sigma\left(u_{k}\right)\right)=\sigma\left(u_{k+1}\right)=\sigma\left(D u_{k}\right)$ for all $k$. In order to conclude that the bijective linear map determined by $\sigma$ is a Lie algebra isomorphism it suffices to check that the defining relations of $S$ are satisfied in $H\left(2: \mathbf{n} ; \omega_{2}\right)$. In fact, we have

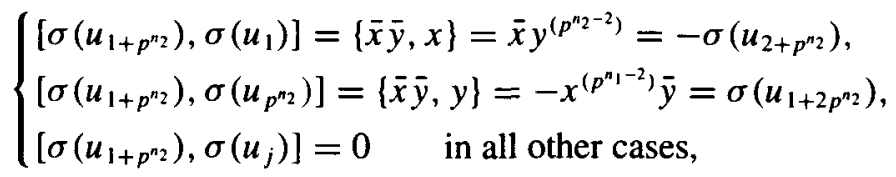

where the last formula holds because $\left\{\bar{x} \bar{y}, x^{(k)} y^{(l)}\right\}=0$ unless $k, l \leq 1$, and also

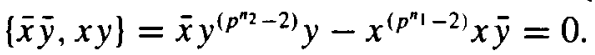

Incidentally, the sequence of the constituent lengths in the graded Lie algebra of maximal class $A F S(0, b, n, p)$, which was computed in [22] and utilized in [24], can now be more easily deduced from the isomorphism $\sigma$. In fact, viewing the two-step centralizers in the corresponding (twisted) loop algebra of $H\left(2: \mathbf{n} ; \omega_{2}\right)$ and setting $q=p^{n_{2}}$ we see at once that all components $L_{k}$ are centralized by $Y=\left(D+u_{1}\right) \otimes t=$ $(D+x) \otimes t$, except when $k \equiv 1(\bmod q-1)$ and $q \not \equiv 1\left(\bmod p^{n}-1\right)$, in which cases $L_{k}$ is centralized by $X=u_{1} \otimes t=x \otimes t$ (in the usual notation of [22] but with capital letters instead).

Another consequence of the isomorphism $\sigma$ is a formula for carrying out explicit computations in the Lie algebra of maximal class $\operatorname{AFS}(0, b, n, p)$. In fact, setting

$$
\left[u_{i q+j}, u_{k q+l}\right]=c(i, j, k, l) \cdot u_{(i+k) q+(j+l)}
$$

for $0<i, k \leq p^{n_{1}}$ and $0<j, l \leq p^{n_{2}}$, with $(i, j)$ and $(k, l) \neq\left(p^{n_{1}}, p^{n_{2}}\right)$, we have

$$
\begin{aligned}
c(i, j, k, l)= & -\left(\begin{array}{c}
2 p^{n_{1}}-i-k-1 \\
p^{n_{1}}-i
\end{array}\right)\left(\begin{array}{c}
2 p^{n_{2}}-j-l-1 \\
p^{n_{2}}-j-1
\end{array}\right) \\
& +\left(\begin{array}{c}
2 p^{n_{1}}-i-k-1 \\
p^{n_{1}}-i-1
\end{array}\right)\left(\begin{array}{c}
2 p^{n_{2}}-j-l-1 \\
p^{n_{2}}-j
\end{array}\right) .
\end{aligned}
$$

This rather unpleasant formula can be put into the slightly simpler form

$$
(-1)^{i+j} \cdot c(i, j, k, l)=\left(\begin{array}{c}
k \\
p^{n_{1}}-i
\end{array}\right)\left(\begin{array}{c}
l-1 \\
p^{n_{2}}-j-1
\end{array}\right)-\left(\begin{array}{c}
k-1 \\
p^{n_{1}}-i-1
\end{array}\right)\left(\begin{array}{c}
l \\
p^{n_{2}}-j
\end{array}\right)
$$

by means of standard binomial coefficient manipulations and the less standard but easily proved fact that the value of $\left(\begin{array}{l}n \\ k\end{array}\right)$ modulo $p$ (for $n, k \in \mathbb{Z}$ ) is periodic in $n$ with period the smallest power of $p$ which is greater than $k$. Since any nonzero homogeneous element of weight $k \geq 2$ of $A F S(0, b, n, p)$ (realized as a loop algebra of $H\left(2:\left(n-n_{2}, n_{2}\right) ; \omega_{2}\right)$ as above $)$ can be uniquely written as a scalar multiple of

$$
\left[Y, X, Z_{3}, Z_{4}, \ldots, Z_{k}\right]=u_{k} \otimes t
$$


with $Z_{i} \in\{X,-Y\}$, the above formula allows one to multiply homogeneous elements in $A F S(0, b, n, p)$ without any need for commutator expansions. Finally, the method of iterated deflation described in [22] reduces computations in $\operatorname{AFS}(a, b, n, p)$ to computations in $A F S(0, b-a, n, p)$.

The isomorphism which we have seen above between the algebra of Albert and Frank $A F(0, b, n, p)$ and the Hamiltonian algebra $H\left(2: \mathbf{n} ; \omega_{2}\right)$ is perhaps better expressed in terms of the basis $\left\{e_{\xi}\right\}$ of $A F(0, b, n, p)$. As we have mentioned above, the general case of $A F(a, b, n, p)$ can be reduced to that of $A F(0, b-a, n, p)$ as in [22], and we record the more general case in the following theorem (which can, of course, also be proved by direct but rather tedious computation).

THEOREM 6.1. The algebra of Albert and Frank $A F(a, b, n, p)$ with Lie bracket given by (6.1) (in arbitrary prime characteristic $p$ ) is isomorphic with the Hamiltonian algebra $H\left(2: \mathbf{n} ; \omega_{2}\right)$, where $\mathbf{n}=(n-b+a, b-a)$. An isomorphism is given by the linear map $\sigma: A F(a, b, n, p) \rightarrow H\left(2: \mathbf{n} ; \omega_{2}\right)$ defined by the formula

$$
\sigma\left(e_{\xi}\right)=\sum \xi^{i p^{b}+j p^{a}} x^{(i)} y^{(j)},
$$

where the summation is over all pairs $(i, j)$ with $0 \leq i<n-b+a, 0 \leq j<b-a$ and $(i, j) \neq(0,0)$. The inverse map is given by

$$
\sigma^{-1}\left(x^{(i)} y^{(j)}\right)=-\sum_{\xi \in F_{p^{n}}^{*}} \xi^{-i p^{b}-j p^{a}} e_{\xi} .
$$

This result gives an explicit realization of $H\left(2: \mathbf{n} ; \omega_{2}\right)$ as a Block algebra. Note that the subalgebra of $A F(a, b, n, p)$ which corresponds to the soluble subalgebra $\left\langle x^{(i)} y^{(j)}: i+j>0\right\rangle$ of $H\left(2: \mathbf{n} ; \omega_{2}\right)$ under the isomorphism $\sigma$ consists of all elements $\sum_{\xi} c_{\xi} e_{\xi}$ with $\sum_{\xi} c_{\xi} \xi^{p^{a}}=\sum_{\xi} c_{\xi} \xi^{p^{b}}=0$. Because of this isomorphism (or by direct verification) this subalgebra of $A F(a, b, n, p)$ is maximal whenever the latter is simple (that is, except when $p=2$ and either $b-a=1$ or $n-b+a=1$ ). It is known that for $p>3$ this is the only maximal subalgebra of codimension two, see [8, Theorem 2.16].

Now we compute the derivation algebra of an algebra of Albert and Frank. According to the identification of the algebras of Albert and Frank with suitable Hamiltonian algebras given in Theorem 6.1, we may consider their derivation algebras as known from the general results on algebras of Cartan type quoted in Section 4, but only for $p>3$. Also, the derivation algebras of all Block algebras (thus including the algebras of Albert and Frank) were computed already in [11], again under the assumption that $p>3$. The reason for this assumption in [11] was that genuine exceptions occur for $p=2,3$ in the more general case of Block algebras. In particular, additional derivations may occur for algebras $H\left(2: \mathbf{n} ; \omega_{0}\right)$ in low characteristics, as we have 
illustrated in Remarks 4.3 and 4.4. Such exceptions do not arise for the algebras of Albert and Frank, that is, for Hamiltonian algebras $H\left(2: \mathbf{n} ; \omega_{2}\right)$. However, Block's proof in [11] (in particular, Lemma 12) was not devised to deal with low characteristics in this special case, and we offer a variation of it which works in every characteristic.

Every specialization of the defining $\mathbb{F}_{p^{n}}$-grading of the algebra of Albert and Frank $A F(a, b, n, p)$ to an $\mathbb{F}_{p}$-grading (this terminology was explained in Section 5) gives rise to a derivation which multiplies each basis element $e_{\xi}$ by its $\mathbb{F}_{p^{-}}$ degree. These derivations turn out to be a basis for the space of outer derivations of $L=A F(a, b, n, p)$. (In fact, these derivations span a maximal torus $T$ in $\operatorname{Der}(L)$, and the given grading is the corresponding decomposition of $L$ into root spaces.)

THEOREM 6.2. The outer derivation algebra $\operatorname{Der}(L) / \operatorname{ad}(L)$ of the algebra of Albert and Frank $A F(a, b, n, p)$ has dimension $n$. More precisely, every derivation of degree zero with respect to the $\mathbb{F}_{p^{n}}$-grading of $L$ acts as $D_{\pi} e_{\xi}=c_{\xi} \cdot e_{\xi}$, for some additive map $\pi: \xi \mapsto c_{\xi}$ of $\mathbb{F}_{p^{n}}$ to itself; all derivations of nonzero degree are inner. Thus a basis for the space of outer derivations is given by the derivations $\left(D_{\mathrm{id}}\right)^{s} e_{\xi}=\xi^{p^{s}} \cdot e_{\xi}$, for $0 \leq s<n$.

PROOF. Clearly $\operatorname{Der}(L)$ inherits an $\mathbb{F}_{p^{n}}$-grading from $L$, and the maps described are derivations of degree zero. Conversely, let $D$ be a derivation of degree zero. Then we have $D e_{\xi}=c_{\xi} \cdot e_{\xi}$ for $\xi \in \mathbb{F}_{p^{n}}^{*}$, for suitable scalars $c_{\xi} \in \mathbb{F}_{p^{n}}$, and we may set $c_{0}=0$. The Leibniz rule $D\left[e_{\xi}, e_{\eta}\right]=\left[D e_{\xi}, e_{\eta}\right]+\left[e_{\xi}, D e_{\eta}\right]$ for $\xi, \eta \in \mathbb{F}_{p^{n}}^{*}$ yields that $c_{\xi+\eta}=c_{\xi}+c_{\eta}$ provided $\left[e_{\xi}, e_{\eta}\right] \neq 0$. The latter amounts to $\xi^{p^{a}} \eta^{p^{b}}-\xi^{p^{b}} \eta^{p^{a}} \neq 0$, which is satisfied as long as $\xi$ and $\eta$ do not span the same $\mathbb{F}_{p^{c}}$-subspace of $\mathbb{F}_{p^{n}}$, where $c=(b-a, n)$. But if $\xi$ and $\eta$ do span the same $\mathbb{F}_{p^{c}}$-subspace of $\mathbb{F}_{p^{n}}$, we may choose $\theta$ outside this subspace and obtain that $c_{\xi+\eta}+c_{\theta}=c_{\xi+\eta+\theta}=c_{\xi+\theta}+c_{\eta}=c_{\xi}+c_{\eta}+c_{\theta}$ (which also settles the case where $\xi+\eta=0$ ). Thus the map $c: \mathbb{F}_{p^{n}} \rightarrow \mathbb{F}_{p^{n}}$ is additive. These maps form an $n$-dimensional $\mathbb{F}_{p^{n}}$-space.

To simplify the calculations which follow we assume $a=0$, as we may, and set $q=p^{b}$. Let $D$ be a derivation of degree $\tau \in \mathbb{F}_{p^{n}}^{*}$, hence $D e_{\xi}=c_{\xi} \cdot e_{\xi+\tau}$ for $\xi \in \mathbb{F}_{p^{n}}^{*}$ (recalling our convention that $e_{0}=0$ ), where $c_{\xi} \in \mathbb{F}_{p^{n}}$, and we may set $c_{0}=c_{-\tau}=0$. Then the condition $D\left[e_{\xi}, e_{\eta}\right]=\left[D e_{\xi}, e_{\eta}\right]+\left[e_{\xi}, D e_{\eta}\right]$ translates into the equation

$$
\left(\xi \eta^{q}-\xi^{q} \eta\right) \cdot c_{\xi+\eta}=\left((\xi+\tau) \eta^{q}-(\xi+\tau)^{q} \eta\right) \cdot c_{\xi}+\left(\xi(\eta+\tau)^{q}-\xi^{q}(\eta+\tau)\right) \cdot c_{\eta}
$$

for $\xi, \eta \in \mathbb{F}_{p^{n}}^{*}$ with $\xi+\eta \neq-\tau$. However, this equation is trivially satisfied also if $\xi=0$ or $\eta=0$ or $\xi+\eta=-\tau$, hence it holds for all $\xi, \eta \in \mathbb{F}_{p^{n}}$.

For the sake of clarity, we only solve the equation in the case where $\tau=1$. This is no loss because in general the equation can be reduced to this case by setting $c_{\xi}^{\prime}=c_{\xi \tau}$. The condition for $D$ being a derivation can be written as

$$
\left(\xi \eta^{q}-\xi^{q} \eta\right)\left(c_{\xi+\eta}-c_{\xi}-c_{\eta}\right)=\left(\eta^{q}-\eta\right) \cdot c_{\xi}+\left(\xi-\xi^{q}\right) \cdot c_{\eta} \quad \text { for } \xi, \eta \in \mathbb{F}_{p^{n}}
$$


One solution is $c_{\xi}=\xi^{q}-\xi$, which corresponds to the inner derivation ad $e_{1}$. We will prove that this is the only solution, up to scalars.

Setting $\eta=-\xi$ in equation (6.2), we obtain that $\left(\xi^{q}-\xi\right)\left(c_{\xi}+c_{-\xi}\right)=0$, hence $c_{-\xi}=-c_{\xi}$ holds provided $\xi^{q}-\xi \neq 0$. Our next goal is to show that the left-hand side of (6.2) always vanishes. We may assume that $\xi^{q}-\xi$ and $\eta^{q}-\eta$ do not both vanish, otherwise the coefficient $\xi \eta^{q}-\xi^{q} \eta$ vanishes, too. Then the left-hand side of (6.2) can also be written as $-\left(\xi \eta^{q}-\xi^{q} \eta\right)\left(c_{-\xi-\eta}+c_{\xi}+c_{\eta}\right)$ if $\xi^{q}+\eta^{q}-\xi-\eta \neq 0$, and as $\left(\xi \eta^{q}-\xi^{q} \eta\right)\left(c_{\xi+\eta}+c_{-\xi}+c_{-\eta}\right)$ otherwise. Either expression is invariant under the cyclic substitution $\xi \mapsto \eta \mapsto-\xi-\eta \mapsto \xi$. Hence the right-hand side of (6.2) is invariant, too, implying that

$$
\begin{aligned}
\left(\eta^{q}-\eta\right) \cdot c_{\xi}-\left(\xi^{q}-\xi\right) \cdot c_{\eta} & =\left(-\xi^{q}-\eta^{q}+\xi+\eta\right) \cdot c_{\eta}-\left(\eta^{q}-\eta\right) \cdot c_{-\xi-\eta} \\
& =\left(\xi^{q}-\xi\right) \cdot c_{-\xi-\eta}-\left(-\xi^{q}-\eta^{q}+\xi+\eta\right) \cdot c_{\xi}
\end{aligned}
$$

or, equivalently,

$$
\left(\xi^{q}-\xi\right)\left(c_{-\xi-\eta}+c_{\xi}+c_{\eta}\right)=\left(\eta^{q}-\eta\right)\left(c_{-\xi-\eta}+c_{\xi}+c_{\eta}\right)=0 .
$$

(A more conceptual view of the computation is the following: invariance of the righthand side of (6.2) under the substitution implies that the (standard) vector product of the two vectors $\left(c_{\xi}, c_{\eta}, c_{-\xi-\eta}\right)$ and $\left(\xi^{q}-\xi, \eta^{q}-\eta,-\xi^{q}-\eta^{q}+\xi+\eta\right)$ is a multiple of $(1,1,1)$; since the vector product is orthogonal to both vectors we have $c_{-\xi-\eta}+c_{\xi}+c_{\eta}=0$, provided the second vector is nonzero.) Consequently, both sides of (6.2) vanish for all $\xi, \eta \in \mathbb{F}_{p^{n}}$. Hence $\left(\eta^{q}-\eta\right) \cdot c_{\xi}=\left(\xi^{q}-\xi\right) \cdot c_{\eta}$, and fixing $\eta$ such that $\eta^{q}-\eta \neq 0$ we conclude that $c_{\xi}=\left(\left(\eta^{q}-\eta\right)^{-1} c_{\eta}\right) \cdot\left(\xi^{q}-\xi\right)$ for all $\xi$.

We have already used Theorem 6.2 in Section 4 to extend the scope of Theorem 4.2 to characteristic three. We mention that a convenient basis for the second cohomology group of $A F(a, b, n, p)$ is obtained (in odd characteristic) from the outer derivations given in Theorem 6.2 by the method explained in Section 4 , and consists of the cocycles $\varphi\left(\left(D_{\text {id }}\right)^{p^{\prime}}\right)\left(e_{\xi}, e_{\eta}\right)=\delta(\xi+\eta, 0) \cdot \xi^{p^{s}}$ for $0 \leq s<n$. In characteristic two these functions are not cocycles because they are not alternating and, in fact, the second cohomology group vanishes, see Remark 4.5.

\section{Another thin grading of $H\left(2: n ; \omega_{2}\right)$}

In this section we introduce $\mathrm{a} \mathbb{Z} /\left(p^{n_{2}}-1\right) \mathbb{Z} \times(\mathbb{Z} / p \mathbb{Z})^{n_{1}}$-grading of $L=H\left(2: \mathbf{n} ; \omega_{2}\right)$. In the special case $n_{1}=1$, the grading will be (cyclic and) thin, and the corresponding loop algebra will be the thin Lie algebra with all the diamonds of finite type constructed in [20]. In fact, the construction of the present grading is guided by the structure of that thin Lie algebra. Unlike in [20], here we put no restriction on the (positive) characteristic. 
To briefly outline the construction of the grading, we first note that such a grading gives rise to a $\mathbb{Z} /\left(p^{n_{2}}-1\right)$-grading and to a $(\mathbb{Z} / p \mathbb{Z})^{n_{1}}$-grading by specialization. Conversely, the original grading can be recovered from these two specializations. We start with the $\mathbb{Z} /\left(p^{n_{2}}-1\right) \mathbb{Z}$-grading of $L$ mentioned at the beginning of Section 5 where $(R, S)=(0,-1)$. Its components are

$$
\bar{L}_{1}=\left\langle x^{(i)} \mid i=1, \ldots, p^{n_{1}}-1\right\rangle+\left\langle x^{(i)} \bar{y} \mid i=0, \ldots, p^{n_{1}}-1\right\rangle,
$$

and

$$
\bar{L}_{1-j}=\left\langle x^{(i)} y^{(j)} \mid i=0, \ldots, p^{n_{1}}-1\right\rangle
$$

for $j=1, \ldots, p^{n_{2}}-2$. The component of degree zero is isomorphic with a Zassenhaus algebra $W\left(1: n_{1}\right)$. As we saw in Section 2, the latter has a grading over the additive group of the field $\mathbb{F}_{p^{n_{1}}}$, which is simply the root space decomposition of $W\left(1: n_{1}\right)$ with respect to an appropriate one-dimensional Cartan subalgebra, say $\left\langle e_{0}\right\rangle$. Viewing $W\left(1: n_{1}\right)$ as a subalgebra of $L$, the decomposition of $L$ into weight spaces with respect to ad $e_{0}$ extends the grading of $W\left(1: n_{1}\right)$ to a grading of $L$, which turns out to be over the same group $\mathbb{F}_{p^{n_{1}}}$. Since $e_{0} \in \bar{L}_{0}$, it normalizes every component $\bar{L}_{j}$, and it follows that this $\mathbb{F}_{p^{n_{1}}}$-grading of $L$ together with the $\mathbb{Z} /\left(p^{n_{2}}-1\right) \mathbb{Z}$-grading yield the

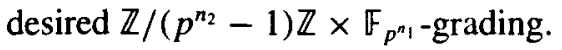

As announced above, we identify the subalgebra

$$
W=\left\langle x^{(i)} y \mid i=0, \ldots, p^{n_{1}}-1\right\rangle
$$

of $L$ with the Zassenhaus algebra $W\left(1: n_{1}\right)$ via $E_{i}=x^{(i+1)} y$, for $i=-1, \ldots, p^{n_{i}}-2$. This is justified by

$$
\left\{x^{(i)} y, x^{(k)} y\right\}=\left(\left(\begin{array}{c}
i+k-1 \\
k-1
\end{array}\right)-\left(\begin{array}{c}
i+k-1 \\
i-1
\end{array}\right)\right) x^{(i+k-1)} y .
$$

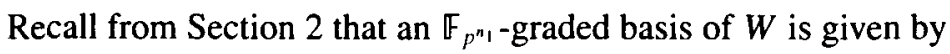

$$
\left\{\begin{array}{l}
e_{0}=y+\bar{x} y \\
e_{\alpha}=\bar{x} y+\sum_{i=0}^{p^{n_{1}-1}} \alpha^{i} x^{(i)} y \quad \text { for } \alpha \in \mathbb{F}_{p^{n}}^{*}
\end{array}\right.
$$

(where the former formula can be seen as a special case of the latter, but we have kept them separated for the sake of clarity, here and below).

It is a simple matter to find the weight spaces of ad $e_{0}$ on each component $\bar{L}_{j}$, starting from the formula

$$
\begin{aligned}
\left\{e_{0}, x^{(i)} y^{(j)}\right\} & =\left\{y+\bar{x} y, x^{(i)} y^{(j)}\right\}= \\
& = \begin{cases}-j x^{\left(p^{1}-2\right)} y^{(j)} & \text { if } i=0, \\
(1+e) y^{(j)}+(1+j) \bar{x} y^{(j)} & \text { if } i=1, \\
x^{(i-1)} y^{(j)} & \text { if } i>1 .\end{cases}
\end{aligned}
$$


In particular, eigenvectors for ad $e_{0}$ on $\bar{L}_{1-j}$, for $j=1, \ldots, p^{n_{2}}-1$, are

$$
\left\{\begin{array}{l}
e_{1-j, 0}=y^{(j)}+j \bar{x} y^{(j)} \\
e_{1-j, \alpha}=j \bar{x} y^{(j)}+\sum_{i=0}^{p^{n_{1}-1}} \alpha^{i} x^{(i)} y^{(j)} \quad \text { for } \alpha \in \mathbb{F}_{p^{n_{1}}}^{*},
\end{array}\right.
$$

extending the complete set of eigenvectors for ad $e_{0}$ on $W$ given by its basis elements $e_{0, \alpha}=e_{\alpha}$. Our notation is chosen so that $e_{1-j, \alpha} \in \bar{L}_{1-j}$, and

$$
\left\{e_{0}, e_{1-j, \alpha}\right\}=\alpha e_{1-j, \alpha} .
$$

Also, the subscript $1-j$ in $e_{1-j, \alpha}$ will be read modulo $p^{n_{2}}-1$, so that, for example, $e_{1, \alpha}$ will be the same as $e_{2-p^{n_{2}, \alpha}}$. However, beware that $e_{1, \alpha}$ is not what one would obtain by putting $j=0$ in the formulas.

The formulas above give complete sets of eigenvectors for ad $e_{0}$ on $\bar{L}_{1-j}$ only for $j=1, \ldots, p^{n_{2}}-2$. In fact, ad $e_{0}$ acts on $\bar{L}_{2-p^{n_{2}}}$ as a sum of two-dimensional Jordan blocks, say $\left\langle e_{1, \alpha}, \bar{e}_{1, \alpha}\right\rangle$, one for each eigenvalue $\alpha \in \mathbb{F}_{p^{n_{1}}}^{*}$. (These will be the diamonds in the thin grading, in case $n_{1}=1$.) It is convenient to assume that

$$
\left\{e_{0}, \bar{e}_{1, \alpha}\right\}=\alpha \bar{e}_{1, \alpha}-\alpha e_{1, \alpha},
$$

which we may because $\alpha \neq 0$. This determines $\bar{e}_{1, \alpha} \in \bar{L}_{1}$ modulo $\left\langle e_{1, \alpha}\right\rangle$, and we choose to set $\bar{e}_{1, \alpha}=\sum_{i=1}^{p^{n_{1}}-1} \alpha^{i}\left(x^{(i)}-i x^{(i)} \bar{y}\right)$ for $\alpha \in \mathbb{F}_{p^{n} \mid}^{*}$. It will be convenient to allow also the value $\alpha=0$ in the above formula, and thus set $\bar{e}_{1,0}=0$. (In case $n_{1}=1$ the component $\left\langle e_{1,0}, \bar{e}_{1,0}\right\rangle=\left\langle e_{1.0}\right\rangle$ corresponds to a fake diamond in the thin grading.)

In the following lemma, we summarize what we have obtained so far, and we include the inverse formulas which give the divided powers in terms of the elements $e_{j, \alpha}$ and $\bar{e}_{j, \alpha}$.

LEMMA 7.1. The Hamiltonian algebra $L=H\left(2: \mathbf{n} ; \omega_{2}\right)$ admits $a \mathbb{Z} /\left(p^{n_{2}}-1\right) \mathbb{Z} \times$

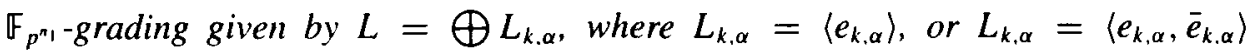
whenever the latter makes sense, and the basis elements involved are given by

$$
\begin{cases}e_{1-j, \alpha}=j \bar{x} y^{(j)}+\sum_{i=0}^{p^{n_{1}}-1} \alpha^{i} x^{(i)} y^{(j)} & \text { for } \alpha \in \mathbb{F}_{p^{n_{1}}} \text { and } j=1, \ldots, p^{n_{2}}-1, \\ \bar{e}_{1, \alpha}=\sum_{i=1}^{p^{n_{1}}-1} \alpha^{i}\left(x^{(i)}-i x^{(i)} \bar{y}\right) & \text { for } \alpha \in \mathbb{F}_{p^{n_{1}}}^{*}\end{cases}
$$

where $0^{0}$ should be understood as 1 . Conversely, for $i=1, \ldots, p^{n_{1}}-1$ and $j=$ $1, \ldots, p^{n_{2}}-1$ we have

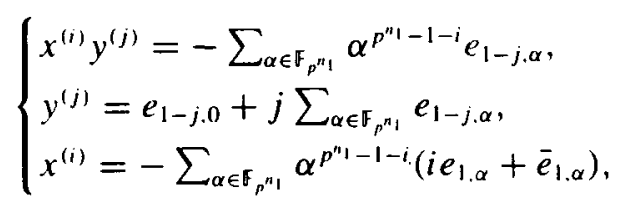

where in the last formula it is understood that $\bar{e}_{1,0}=0$. 
For later use, we record the products of the basis elements given in Lemma 7.1. We have

$$
\left\{e_{1-j, \alpha}, e_{1-l, \beta}\right\}=\left(\beta\left(\begin{array}{c}
j+l-1 \\
l
\end{array}\right)-\alpha\left(\begin{array}{c}
j+l-1 \\
j
\end{array}\right)\right) e_{2-j-l, \alpha+\beta}
$$

for $j, l=1, \ldots, p^{n_{2}}-1$, provided $1 \leq j+l-1 \leq p^{n_{2}}-1$, and zero otherwise,

$$
\begin{aligned}
& \left\{e_{1-j, \alpha}, \bar{e}_{1, \beta}\right\}=\beta e_{2-j, \alpha+\beta} \quad \text { for } j=2, \ldots, p^{n_{2}}-1, \\
& \left\{e_{0, \alpha}, \bar{e}_{1, \beta}\right\}=-\beta e_{1, \alpha+\beta}+\beta \bar{e}_{1, \alpha+\beta}, \\
& \left\{\bar{e}_{1, \alpha}, \bar{e}_{1, \beta}\right\}=0 .
\end{aligned}
$$

It is not necessary to carry out the computations in full in order to prove these formulas, if we use the fact that the basis is graded, according to Lemma 7.1. For example, the result of the product in formula (7.1) must be a scalar multiple of $e_{2-j-l, \alpha+\beta}$, and we only have to find that scalar. Since the monomial $y^{(j)}$ appears in $e_{1-j, \alpha}$ with coefficient 1 , it is enough to compute the coefficient of the monomial $y^{(j+l-1)}$ in the product $\left\{e_{1-j, \alpha}, e_{1-l, \beta}\right\}$. The products of the only relevant terms, namely

$$
\left\{y^{(j)}, \beta x y^{(l)}\right\}+\left\{\alpha x y^{(j)}, y^{(l)}\right\}=\left(\beta\left(\begin{array}{c}
j+l-1 \\
l
\end{array}\right)-\alpha\left(\begin{array}{c}
j+l-1 \\
j
\end{array}\right)\right) y^{(j+l-1)},
$$

yield the desired conclusion. Formula (7.2) follows similarly by computing

$$
\left\{y^{(j)}, \beta x\right\}=\beta y^{(j-1)} \text {. }
$$

Formula (7.4) follows because the monomial $y^{\left(p^{n_{2}}-2\right)}$ does not appear in the result. The proof of formula (7.3) is just slightly more involved because $\left\{e_{0, \alpha}, \bar{e}_{1, \beta}\right\}$ must be a linear combination of $e_{1, \alpha+\beta}$ and $\bar{e}_{1, \alpha+\beta}$. Since the coefficients of $\bar{y}$ and $x$ are 1 and 0 in the former, and 0 and $\alpha+\beta$ in the latter, the conclusion follows by computing the only relevant products, which are $\{y,-\beta x \bar{y}\}=-\beta \bar{y}$ and

$$
\left\{y, \beta^{2} x^{(2)}\right\}+\{\alpha x y, \beta x\}=\beta(\alpha+\beta) x .
$$

(For the dubious case where $\alpha+\beta=0$, recall from Lemma 7.1 that we have set $\bar{e}_{1.0}=0$.)

Now we restrict our attention to the case of main interest for us by letting $n_{1}=1$, and introducing the shorthand $q=p^{n_{2}}$, so that $L$ has dimension $p q-1$. Then the subspaces $L_{k, \alpha}$ form a grading of $L$ over the cyclic group $\mathbb{Z} /(q-1) \mathbb{Z} \times \mathbb{F}_{p}$. Choosing $(1,1)$ as a generator of the latter, we will show that the grading is thin, according to Definition 5.3, and that all diamonds are of finite type. 
The diamonds occur in all degrees congruent to 1 modulo $q-1$, with the exception of degree $q$. We set

$$
X=\bar{e}_{1,1}=\sum_{i=1}^{p-1}\left(x^{(i)}-i x^{(i)} \bar{y}\right) \quad \text { and } \quad Y=e_{1,1}=-\bar{x} \bar{y}+\sum_{i=0}^{p-1} x^{(i)} \bar{y},
$$

and check that the covering property is satisfied. Since

$$
\begin{aligned}
& \left\{\bar{e}_{1, \alpha}, X\right\}=0, \\
& \left\{e_{0, \alpha}, X\right\}=\bar{e}_{1, \alpha+1}-e_{1, \alpha+1} \quad\left(\text { where } \bar{e}_{1,0}=0\right), \\
& \left\{e_{1-j, \alpha}, X\right\}=e_{2-j, \alpha+1} \quad \text { for } j=2, \ldots q-1, \\
& \left\{\bar{e}_{1, \alpha}, Y\right\}=-\alpha e_{2, \alpha+1}, \\
& \left\{e_{0, \alpha}, Y\right\}=(\alpha+1) e_{1, \alpha+1} \\
& \left\{e_{1-j, \alpha}, Y\right\}=0 \text { for } j=2, \ldots q-1,
\end{aligned}
$$

we have $\left\{L_{k, \alpha},\langle X, Y\rangle\right\}=L_{k+1, \alpha+1}$ for $k \not \equiv 1(\bmod (q-1))$, which shows that the covering property holds in these degrees, since $L_{k, \alpha}$ is one-dimensional. To see that the loop algebra is thin, it remains to check the covering property on the two-dimensional components. At the same time we will check the diamond types (including the fake diamond $L_{1,0}$, which is in degree $q$ ). Since

$$
\begin{aligned}
& \left\{e_{0 . \alpha}, X, X\right\}=\left\{-e_{1, \alpha+1}, X\right\}=-e_{2 . \alpha+2}, \\
& \left\{e_{0, \alpha}, X, Y\right\}=\left\{\bar{e}_{1, \alpha+1}, Y\right\}=-(\alpha+1) e_{2, \alpha+2}, \\
& \left\{e_{0, \alpha}, Y, X\right\}=\left\{(\alpha+1) e_{1, \alpha+1}, X\right\}=(\alpha+1) e_{2, \alpha+2}, \\
& \left\{e_{0, \alpha}, Y, Y\right\}=0
\end{aligned}
$$

if $a, b$ are scalars we have $\left\{e_{0, \alpha}, a X+b Y,\langle X, Y\rangle\right\}=L_{2, \alpha+2}$ unless $a=b=0$ in case $\alpha \neq-1$, and unless $a=0$ in case $\alpha=-1$ (the case of the fake diamond). It follows that the grading is thin. Furthermore, the elements just above the (possibly fake) diamonds are those of the form $V_{\alpha}=e_{0,-\alpha-1}$ and satisfy

$$
\left\{V_{\alpha}, Y, X\right\}=\alpha\left\{V_{\alpha}, X, X\right\},
$$

hence the diamond $\left\langle\left\{V_{\alpha}, X\right\},\left\{V_{\alpha}, Y\right\}\right\rangle$ in degree $q+\alpha(q-1)$ (which is fake for $\alpha=0$ ) has type $\alpha$. Note that most of the computations done in this paragraph need not be carried out in full, provided we use the fact that the elements $e_{k, \alpha}$ and $\bar{e}_{1, \alpha}$ form a graded basis of $L$, according to Lemma 7.1.

We have completed the proof of the following result.

THEOREM 7.2. In case $n_{1}=1$ the cyclic grading of $L=H\left(2: \mathbf{n} ; \omega_{2}\right)$ defined in Lemma 7.1 is a thin grading (with respect to the generator $(1,1)$ of the grading group). The diamond types are all finite, and follow an arithmetic progression. 
Note that $\left\{L_{k, \alpha}, Y\right\}=0$ for $k \not \equiv 0,1(\bmod (q-1))$, and also $\left\{L_{0 .-1}, Y\right\}=$ $\left\{L_{1,0}, Y\right\}=0$; hence $Y$ centralizes all one-dimensional components which do not immediately precede a diamond.

We remark that thin Lie algebras with diamonds of both finite and infinite types are constructed in [5]. The finite diamond types there follow an arithmetic progression, but they are separated by sequences of constant length of diamonds of infinite type.

\section{Another realization of $H\left(2:\left(1, n_{2}\right) ; \omega_{2}\right)$ as a Block algebra}

In [20] we constructed a simple Lie algebra $A$ with a thin grading and all diamonds of finite type. Since the location and types of the diamonds of $A$ match those of the algebra $L=H\left(2:\left(1, n_{2}\right) ; \omega_{2}\right)$ with the grading defined in the preceding section it follows that the loop algebras of $L$ and $A$ are isomorphic. Actually, this yields an isomorphism between $L$ and $A$ themselves, but it may be necessary to extend the ground field for this. The purpose of this section is to justify these claims.

We recall the definition of $A$ from [20], with slight notational changes to avoid clash with the notation of the present paper. Let $p$ be any prime, let $q=p^{n_{2}}$, and set $S^{*}=\left(\mathbb{F}_{p} \times \mathbb{F}_{q}\right) \backslash\{(0,0)\}$. The $\mathbb{F}_{q}$-vector space $A$ with basis $\left\{f_{u, \alpha} \mid(u, \alpha) \in S^{*}\right\}$ becomes a Lie algebra over $\mathbb{F}_{q}$ by defining $\left[f_{u, \alpha}, f_{v, \beta}\right]=(v \alpha-u \beta) \cdot f_{u+v, \alpha+\beta}$, where we read $0 \cdot f_{0,0}$ as zero. By construction $A$ is a Block algebra, and so it is simple and isomorphic to a Hamiltonian algebra $H\left(2: \mathbf{n} ; \omega_{2}\right)$ (at least when $p>3$, and possibly after extending the ground field, see Section 3 ). We will construct an isomorphism explicitly.

It was shown in [20] that $A$ has a thin grading where the component of degree one is spanned by $x=f_{1,0}$ and $y=\sum_{\alpha \in \mathbb{F}_{q}} f_{1, \alpha}$. (The assumption $p>3$ stated in [20] was only needed in later sections, when considering presentations for the thin algebra.) An easy induction showed that

$$
[y, \underbrace{x, \ldots, x}_{j-1}]=\sum_{\alpha \in \mathbb{F}_{4}} \alpha^{j-1} f_{j, \alpha} \quad \text { for } j \geq 1
$$

(where our convention that $0^{\circ}=1$ intervenes when $j=1$ ). This element spans the component of degree $j$ of the grading, except when $j \equiv 1(\bmod q-1)$ but $j \not \equiv 0$ $(\bmod p)$, in which cases the component is two-dimensional. An element just above a diamond of type $\lambda-1$ is

$$
v_{\lambda-1}=[y, \underbrace{x, \ldots, x}_{\lambda(q-1)-1}]=\sum_{\alpha \in \mathbb{F}_{4}^{*}} \alpha^{-1} f_{-\lambda, \alpha},
$$

whence

$$
[y, \underbrace{x, \ldots, x}_{\lambda(q-1)-1}, y]=(\lambda-1) \sum_{\gamma \in \mathbb{E}_{4}} f_{-\lambda+1, \gamma} .
$$


In particular, we have

$$
\left\{\begin{array}{l}
{[y, \underbrace{x, \ldots, x}_{p(q-1)}]=\sum_{\alpha \in \mathbb{F}_{q}} \alpha^{(q-1) p} f_{1+(q-1) p, \alpha}=\sum_{\alpha \in \mathbb{F}_{q}^{*}} f_{1, \alpha}=y-x,} \\
{[y, \underbrace{x, \ldots, x}_{p(q-1)-1}, y]=-\sum_{\gamma \in \mathbb{F}_{q}} f_{1, \gamma}=-y .}
\end{array}\right.
$$

Now we carry out analogous computations in $L=H\left(2:\left(1, n_{2}\right) ; \omega_{2}\right)$ with the thin grading constructed in the previous section, using the formulas obtained there. In particular, the component of degree one in the grading is spanned by $X=\bar{e}_{1,1}$ and $Y=e_{1,1}$, and we find that

$$
\{Y, \underbrace{X, \ldots, X\}}_{j-1}\}=(-1)^{\left\lfloor\frac{j-1}{q-1}\right\rfloor}\left(e_{j, j}-\delta_{q-1}(j, 1) \bar{e}_{j, j}\right) \quad \text { for } j>1
$$

(but not for $j=1)$, where $\delta_{q-1}(s, t)$ equals one if $s \equiv t(\bmod q-1)$, and zero otherwise. An element just above a diamond of type $\lambda-1$ is

$$
V_{\lambda-1}=\{Y, \underbrace{X, \ldots, X}_{\lambda(q-1)-1}\}=(-1)^{\lambda-1} e_{0,-\lambda},
$$

whence

$$
\{Y, \underbrace{X, \ldots, X}_{\lambda(q-1)-1}, Y\}=(-1)^{\lambda}(\lambda-1) e_{1,-\lambda+1}
$$

In particular, we have

$$
\left\{\begin{array}{l}
\{Y, \underbrace{X, \ldots, X}_{p(q-1)}\}=-e_{1,1}+\bar{e}_{1,1}=-Y+X, \\
\{Y, \underbrace{X, \ldots, X}_{p(q-1)-1}, Y\}=e_{1,1}=Y .
\end{array}\right.
$$

The sign discrepancy of the latter formulas with the analogous ones for $A$ seen above means nothing if we work with the loop algebras of $A$ and $L$ (which are isomorphic), but suggests that $A$ may not be isomorphic with $L=H\left(2:\left(1, n_{2}\right) ; \omega_{2}\right)$ over $\mathbb{F}_{q}$. We extend the ground field to $\mathbb{F}_{q^{2}}$ and fix $\varepsilon \in \mathbb{F}_{q^{2}}$ with $\varepsilon^{q-1}=-1$. A well-defined linear map $\tau: A \rightarrow L$ is obtained by setting $\tau(x)=\varepsilon X, \tau(y)=\varepsilon Y$, and

$$
\left\{\begin{array}{l}
\tau([y, \underbrace{x, \ldots, x}_{j-2}, x])=\varepsilon^{j}\{Y, \underbrace{X, \ldots, X}_{j-2}, X\} \\
\tau([y, \underbrace{x, \ldots, x}_{j-2}, y])=\varepsilon^{j}\{Y, \underbrace{X, \ldots, X}_{j-2}, Y\}
\end{array}\right.
$$


for all $j \geq 2$. The computations done in Section 7 and in [20] show that $y$ and $Y$ centralize all one-dimensional components which do not immediately precede a diamond, in the respective algebras, and also that the diamond types match. It follows that $\tau([w, z])=\{\tau(w), \tau(z)\}$ for all $w \in A$ and $z=x$ or $y$. Since $x$ and $y$ generate $A$ we conclude that the map $\tau$ is an isomorphism of Lie algebras (because the set of $z \in A$ such that $\tau([w, z])=\{\tau(w), \tau(z)\}$ for all $w \in A$ is a subalgebra).

Finding explicit formulas for the isomorphism $\tau$ in terms of convenient bases for $A$ and $L$ is a matter of some manipulations, and we only record the final result.

THEOREM 8.1. The Block algebra A defined in [20] and described at the beginning of this section is isomorphic with the Hamiltonian algebra $H\left(2:\left(1, n_{2}\right) ; \omega_{2}\right)$. An isomorphism is given by the linear map $\tau: A \rightarrow H\left(2:\left(1, n_{2}\right) ; \omega_{2}\right)$ defined by the formulas (both for $\alpha \in \mathbb{F}_{p}$, and where $\varepsilon^{q-1}=-1$ )

$$
\left\{\begin{array}{l}
\tau\left(f_{\alpha, \beta}\right)=-\sum_{k=1}^{q-1} \beta^{-k+1} \varepsilon^{k} e_{k, \alpha}+\varepsilon \bar{e}_{1, \alpha} \quad \text { for } \beta \in \mathbb{F}_{q}^{*}, \\
\tau\left(f_{\alpha, 0}\right)=\varepsilon \bar{e}_{1, \alpha},
\end{array}\right.
$$

in terms of the basis $\left\{e_{k, \alpha}, \bar{e}_{1, \alpha}\right\}$ of $H\left(2:\left(1, n_{2}\right) ; \omega_{2}\right)$ defined in Lemma 7.1, or

$$
\left\{\begin{array}{l}
\tau\left(f_{\alpha, \beta}-f_{\alpha, 0}\right)=\sum_{j=1}^{q-1} j \varepsilon^{1-j} \beta^{j} \bar{x} y^{(j)}+\sum_{i=0}^{p-1} \sum_{j=1}^{q-1} \varepsilon^{1-j} \alpha^{i} \beta^{j} x^{(i)} y^{(j)} \quad \text { for } \beta \in \mathbb{F}_{q}^{*}, \\
\tau\left(f_{\alpha, 0}\right)=\varepsilon \sum_{i=1}^{p-1} \alpha^{i}\left(x^{(i)}-i x^{(i)} \bar{y}\right)
\end{array}\right.
$$

in terms of divided powers.

Note that there appears to be a certain amount of symmetry between the formulas for $\tau\left(f_{0, \beta}\right)$ and $\tau\left(f_{\alpha, 0}\right)$, because $\tau\left(f_{0, \beta}\right)=\sum_{j=1}^{q-1} \varepsilon^{1-j} \beta^{j}\left(y^{(j)}+j \bar{x} y^{(j)}\right)$.

The following cocycles of $A$ were introduced in [20], and it was proved that they form a basis for the second cohomology group of $A$, if $p>3$

$$
\varphi_{r}\left(f_{u, \alpha}, f_{v, \beta}\right)= \begin{cases}\alpha^{p^{\prime}} & \text { if } \alpha+\beta=0 \text { and } u+v=0 \\ 0 & \text { otherwise }\end{cases}
$$

for $1 \leq r \leq n_{2}$, and

$$
\psi\left(f_{u, \alpha}, f_{v, \beta}\right)= \begin{cases}u & \text { if } u+v=0 \\ 0 & \text { otherwise }\end{cases}
$$

In view of the isomorphism $\tau$ given in Theorem 8.1, we now make the connection with the cocycles of $L=H\left(2:\left(1, n_{2}\right) ; \omega_{2}\right)$ described in Theorem 4.2. Call $\Phi_{r}$ and $\Psi$ the cocycles obtained by pulling the cocycles $\varphi_{r}$ and $\psi$ of $A$ back to $L$ via the isomorphism $\tau^{-1}$. A straightforward computation for $\Psi$, and a slightly more involved one for $\Phi_{r}$, both of which we omit, show that $\Psi=-\varepsilon^{-2} \psi_{1}$ and that

$$
\Phi_{r}=\varepsilon^{p^{r}-2}\left(\varphi_{r}+\varphi\left(\operatorname{ad} x y^{\left(q-p^{r}\right)}\right)\right)
$$

in the notation of Section 4. 


\section{Acknowledgement}

We are grateful to the referees for their comments.

\section{References}

[1] A. A. Albert and M. S. Frank, 'Simple Lie algebras of characteristic p', Univ. e Politec. Torino. Rend. Sem. Mat. 14 (1954-55), 117-139.

[2] M. Avitabile, Some loop algebras of Hamiltonian Lie algebras (Ph.D. Thesis, Trento, November 1999).

[3] _ - 'Some loop algebras of Hamiltonian Lie algebras', Internat. J. Algebra Comput. (4) 12 (2002), 535-567.

[4] M. Avitabile and G. Jurman, 'Diamonds in thin Lie algebras', Boll. Unione Mat. Ital. Sez. B Artic. Ric. Mat. (8) (3) 4 (2001), 597-608.

[5] M. Avitabile and S. Mattarei, 'Thin Lie algebras with diamonds of finite and infinite type', $J$. Algebra 293 (2005), 34-64.

[6] — 'Thin loop algebras of Albert-Zassenhaus algebras', preprint.

[7] G. Benkart, A. I. Kostrikin and M. I. Kuznetsov, 'Finite-dimensional simple Lie algebras with a nonsingular derivation', J. Algebra (3) 171 (1995), 894-9l6.

[8] G. Benkart and J. M. Osborn, 'Toral rank one Lie algebras', J. Algebra (1) 115 (1988), 238-250.

[9] G. M. Benkart, T. B. Gregory, J. M. Osborn, H. Strade and R. L. Wilson, 'Isomorphism classes of Hamiltonian Lie algebras', in: Lie algebras, Madison 1987, Lecture Notes in Math. 1373 (Springer, Berlin, 1989) pp. 42-57.

[10] G. M. Benkart and R. V. Moody, 'Derivations, central extensions, and affine Lie algebras', Algebras Groups Geom. (4) 3 (1986), 456-492.

[11] R. Block, 'New simple Lie algebras of prime characteristic', Trans. Amer. Math. Soc. 89 (1958), $421-449$.

[12] R. E. Block, 'Determination of the differentiably simple rings with a minimal ideal.' Ann. of Math. (2) 90 (1969), 433-459.

[13] R. E. Block and R. Lee Wilson, 'The simple Lie p-algebras of rank two', Ann. of Math. (2) (1) 115 (1982), 93-168.

[14] R. Brandl, 'The Dilworth number of subgroup lattices', Arch. Math. (Basel) (6) 50 (1988), 502-510.

[15] R. Brandl, A. Caranti and C. M. Scoppola, 'Metabelian thin p-groups', Quart. J. Math. Oxford Ser. (2) (170) 43 (1992), 157-173.

[16] A. Caranti, 'Presenting the graded Lie algebra associated to the Nottingham group', J. Algebra (1) 198 (1997), 266-289.

[17] _ - 'Thin groups of prime-power order and thin Lie algebras: an addendum', Quart. J. Math. Oxford Ser. (2) (196) 49 (1998), 445-450.

[18] —_ 'Loop algebras of Zassenhaus algebras in characteristic three', Israel J. Math. 110 (1999), $61-73$.

[19] A. Caranti and G. Jurman, 'Quotients of maximal class of thin Lie algebras. The odd characteristic case', Comm. Algebra (12) 27 (1999), 5741-5748.

[20] A. Caranti and S. Mattarei, 'Some thin Lie algebras related to Albert-Frank algebras and algebras of maximal class', J. Austral. Math. Soc. Ser. A (2) 67 (1999), 157-184 Group theory.

[21] _ 'Nottingham Lie algebras with diamonds of finite type', Internat. J. Algebra Comput. (1) 14 (2004), 35-67. 
[22] A. Caranti, S. Mattarei and M. F. Newman, 'Graded Lie algebras of maximal class', Trans. Amer. Math. Soc. (10) 349 (1997), 4021-4051.

[23] A. Caranti, S. Mattarei, M. F. Newman and C. M. Scoppola, 'Thin groups of prime-power order and thin l.ie algebras', Quart. J. Math. Oxford Ser. (2) (187) 47 (1996), 279-296.

[24] A. Caranti and M. F. Newman, 'Graded Lie algebras of maximal class. II', J. Algebra (2) 229 (2000), 750-784.

[25] C. Carrara, '(Finite) presentations of the Albert-Frank-Shalev Lie algebras', Boll. Unione Mat. Ital. Sez. B Artic. Ric. Mat. (8) (2) 4 (2001), 391-427.

[26] A. S. Dzhumadil'daev, 'Central extensions and invariant forms of Lie algebras of positive characteristic of Cartan types', Funktsional. Anal. i Prilozhen. (4) 18 (1984), 77-78.

[27] _ - 'Central extensions of the Zassenhaus algebra and their irreducible representations', Mat. Sb. (N.S.) (4) 126(168) (1985), 473-489, 592.

[28] R. Farnsteiner, 'The associative forms of the graded Cartan type Lie algebras', Trans. Amer. Math. Soc. (1) 295 (1986), 417-427.

[29] _ - 'Central extensions and invariant forms of graded Lie algebras', Algebras Groups Geom. (4) 3 (1986), 431-455.

[30] — 'Dual space derivations and $H^{2}(L, F)$ of modular Lie algebras', Canad. J. Math. (5) 39 (1987), 1078-1106.

[31] G. Jurman, 'Quotients of maximal class of thin Lie algebras. The case of characteristic two', Comm. Algebra (12) 27 (1999), 5749-5789.

[32] _ 'A family of simple Lie algebras in characteristic two', J. Algebra (2) 271 (2004), 454-481.

[33] _ _ 'Graded Lie algebras of maximal class. III', J. Algebra (2) 284 (2005), 435-461.

[34] G. Jurman and D. S. Young, 'Quotients of maximal class of thin Lie algebras in characteristic two: errata and addendum', preprint.

[35] V. G. Kac, 'A description of the filtered Lie algebras with which graded Lie algebras of Cartan type are associated', Izv. Akad. Nauk SSSR Ser. Mat. 38 (1974), 800-834.

[36] G. Klaas, C. R. Leedham-Green and W. Plesken, Linear pro-p-groups of finite width, Lecture Notes in Math. 1674 (Springer, Berlin, 1997).

[37] A. I. Kostrikin, 'The beginnings of modular Lie algebra theory', in: Group theory, algebra, and number theory (Saarbrïcken, 1993) (de Gruyter, Berlin, 1996) pp. 13-52.

[38] A. I. Kostrikin and M. I. Kuznetsov, 'On the structure of modular Lie algebras associated with analytic pro-p-groups', Dokl. Akad. Nauk (5) 339 (1994), 591-593.

[39] _ _ 'Finite-dimensional Lie algebras with a nonsingular derivation', in: Algebra and analysis (Kazan, 1994) (de Gruyter, Berlin, 1996) pp. 81-90.

[40] A. I. Kostrikin and I. R. Šafarevič, 'Graded Lie algebras of finite characteristic', Izv. Akad. Nauk SSSR Ser. Mat. 33 (1969), 251-322.

[41] M. I. Kuznetsov, 'Truncated induced modules over transitive Lie algebras of characteristic $p$ ', Izv. Akad. Nauk SSSR Ser. Mat. (3) 53 (1989), 557-589, 671.

[42] C. R. Leedham-Green, 'The structure of finite p-groups', J. London Math. Soc. (2) (1) 50 (1994), $49-67$.

[43] C. R. Leedham-Green and S. McKay, 'On the classification of $p$-groups of maximal class', Quart. J. Math. Oxford Ser. (2) (139) 35 (1984), 293-304.

[44] - The structure of groups of prime power order, London Math. Soc. Monogr. 27, Oxford Science Publications (Oxford University Press, Oxford, 2002).

[45] C. R. Leedham-Green and M. F. Newman, 'Space groups and groups of prime-power order. I', Arch. Math. (Basel) (3) 35 (1980), 193-202.

[46] È. Lucals, 'Sur les congruences des nombres eulériens et des coefficients différentiels des fonctions trigonométriques, suivant un module premier', Bull. Soc. Math. France 6 (1878), 49-54.

[47] S. Mattarei, 'Some thin pro-p-groups', J. Algebra (1) 220 (1999), 56-72. 
[48] G. B. Seligman, Modular Lie algebras, Ergebnisse der Mathematik und ihrer Grenzgebiete, Band 40 (Springer, New York, 1967).

[49] S. Serconek and R. L. Wilson, 'Classification of forms of restricted simple Lie algebras of Cartun type', Comm. Algebra (6) 19 (1991), 1603-1628.

[50] A. Shalev, 'Simple Lie algebras and Lie algebras of maximal class', Arch. Math. (Basel) (4) 63 (1994), 297-301.

[51] _ 'The structure of finite p-groups: effective proof of the coclass conjectures', Invent. Math. (2) 115 (1994), 315-345.

[52] A. Shalev and E. I. Zelmanov, 'Pro-p groups of finite coclass', Math. Proc. Cambridge Philos. Soc. (3) 111 (1992), 417-421.

[53] S. Skryabin, 'Toral rank one simple Lie algebras of low characteristics', J. Algebra (2) 200 (1998), 650-700.

[54] S. M. Skryabin, 'Classification of Hamiltonian forms over algebras of divided powers', Mat. Sb. (1) 181 (1990), 114-133.

[55] H. Strade, 'Representations of the $\left(p^{2}-1\right)$-dimensional Lie algebras of R. E. Block', Canad. J. Math. (3) 43 (1991), 580-616.

[56] _ _ 'The classification of the simple modular Lie algebras. VI. Solving the final case', Trans. Amer. Math. Soc. (7) 350 (1998), 2553-2628.

[57] _- Simple Lie algebras over fields of positive characteristic. I, volume 38 of de Gruyter Expositions in Mathematics, Structure theory (Walter de Gruyter \& Co., Berlin, 2004).

[58] H. Strade and R. Farnsteiner, Modular Lie algebras and their representations, volume 116 of Monographs and Textbooks in Pure and Applied Mathematics (Marcel Dekker Inc., New York, 1988).

[59] W. L. J. van der Kallen, Infinitesimally central extensions of Chevalley groups, Lecture Notes in Mathematics, Vol. 356 (Springer, Berlin, 1973).

[60] W. C. Waterhouse, 'Automorphisms and twisted forms of generalized Witt Lie algebras', Trans. Amer. Math. Soc. (1) 327 (1991), 185-200.

[61] R. L. Wilson, 'Simple Lie algebras of type S', J. Algebra (2) 62 (1980), 292-298.

[62] P. Zusmanovich, 'Central extensions of current algebras', Trans. Amer. Math. Soc. (1) 334 (1992), 143-152.

Dipartimento di Matematica

Università degli Studi di Trento

via Sommarive 14

I-38050 Povo (Trento)

Italy

e-mail: caranti@science.unitn.it,mattarei@science.unitn.it 